\title{
Necessary Conditions for Existence of Non-Degenerate Hamiltonian Structures
}

\section{Oleg I. Bogoyavlenskij ${ }^{\star}$}

Department of Mathematics and Statistics, Queen's University, Kingston, Canada, K7L 3N6

Received: 28 July 1993 / in revised form: 6 October 1993

\begin{abstract}
The necessary criteria are pointed out for the existence of Hamiltonian and bi-Hamiltonian non-degenerate structures for a nonlinear system of partial differential equations of first order. The results are formulated in terms of the new invariants of the intrinsic geometry, introduced in this paper, connected with the Nijenhuis and Haantjes tensors of a $(1,1)$ tensor field.
\end{abstract}

\section{Introduction}

This paper is devoted to the investigation of the intrinsic geometry of systems of nonlinear partial differential equations of first order

$$
u_{t}^{i}=\sum_{j=1}^{n} A_{j}^{l}\left(u^{1}, \ldots, u^{n}\right) u_{y}^{j}
$$

As it is known, systems (1.1) arise in numerous classical problems of gas dynamics and mathematical physics $[1-4,7-9,12-15]$.

Riemann pointed out in his classical work [1] that the system (1.1) is closely connected with the $(1,1)$ tensor field $A_{j}^{l}\left(u^{1}, \ldots, u^{n}\right)$ defined on the Euclidean space $R^{n}$ with the coordinates $u^{1}, \ldots, u^{n}$.

Geometry of the vector fields of eigenvectors of the operators $A_{j}^{i}\left(u^{1}, \ldots, u^{n}\right)$ has been studied in famous papers by Nijenhuis [5] and Haantjes [6].

Hamiltonian systems (1.1) and the associated structures of the Poisson brackets were investigated in $[7-9,14,15]$ along with their applications to the theory of the Whitham equations.

Tensor fields $A_{j}^{i}\left(u^{1}, \ldots, u^{n}\right)$ were considered in $[5,6]$ as vector-valued differential 1 -forms and also as fields of operators defined on the tangent bundle $T\left(M^{n}\right)$. The Nijenhuis tensor $N_{j k}^{i}\left(u^{1}, \ldots, u^{n}\right)$ and the Haantjes tensor $H_{j k}^{i}\left(u^{1}, \ldots, u^{n}\right)$ were considered as the vector-valued differential 2-forms.

\footnotetext{
* Supported by NSERC grant OGPIN 337
} 
We consider in this paper the Nijenhuis and Haantjes tensors as the laws of alternating bilinear multiplications in the tangent bundle. Therefore the Nijenhuis and Haantjes tensors define on the tangent bundle $T\left(M^{n}\right)$ the deformations of the structures of non-associative algebras, which appear to be Lie algebras for several important systems (1.1). This point of view leads us to the definition of the analogues of the Cartan-Killing form on the tangent bundle $T\left(M^{n}\right)$, countable sets of differential 1-forms $\omega_{k}$ and 2-forms $\alpha_{k}$ and $\beta_{k}$ and $(1,3)$ tensors $J_{N}$ and $J_{H}$ which we name the Jacobi tensors. These $(1,3)$ tensors characterize the deviation of the algebraic structures defined by the Nijenhuis and Haantjes tensors from the Lie algebra structures. We define also invariant polynomials $P_{N}(v)$ and $P_{H}(v)$ on the tangent bundle and the associated fibrations of the algebraic submanifolds $V_{N}$ and $V_{H}$ embedded into the tangent bundle $T\left(M^{n}\right)$.

In terms of these constructions we point out several necessary criteria for the existence of non-degenerate Hamiltonian or bi-Hamiltonian structures for a system (1.1). For example, if system (1.1) has a non-degenerate Hamiltonian structure then the invariant polynomials $P_{H}(v)$ are even, $P_{H}(v)=P_{H}(-v)$ and the fibration of the algebraic submanifolds $V_{H}$ is invariant under the involution $v \rightarrow-v$. For $n=4$ existence of a non-degenerate Hamiltonian structure implies that the Haantjes tensor $H(u, v)$ defines a deformation of Lie algebra structures in the tangent bundle $T\left(M^{4}\right)$. For $n=3$ these Lie algebras have to be either simple or commutative. The corresponding Cartan-Killing form $(u, v)_{H}$ defines the metric on the manifold $M^{3}$ that has to be conformally flat and therefore has to satisfy the classical WeylSchouten equations.

The existence of two non-degenerate Hamiltonian structures implies that the Haantjes tensor is reducible and is necessarily zero if the two structures are in general position. In the last case the Hamiltonian system possesses the Riemann invariants and is integrable by the generalized hodograph transformation.

These necessary criteria can be checked by a direct calculation of the Haantjes tensor for any system of partial differential equations (1.1).

The efficiency of the geometric methods of this paper is demonstrated for the equations of classical gas dynamics, for the perturbations of the Benney equations and for the certain matrix partial differential equations of the form (1.1).

\section{Gauge Invariance of the Haantjes Tensor}

I. Let $\tilde{u}$ and $\tilde{v}$ be two vector fields on a manifold $M^{n}$ with a $(1,1)$ tensor field $A_{j}^{i}$. Let $u$ and $v$ are values of the vector fields $\tilde{u}$ and $\tilde{v}$ in a point $P$. The Nijenhuis tensor $N(u, v)[5]$ is defined by the formula

$$
N(u, v)=A^{2}[\tilde{u}, \tilde{v}]+[A \tilde{u}, A \tilde{v}]-A([A \tilde{u}, \tilde{v}]+[\tilde{u}, A \tilde{v}])
$$

where $[\tilde{x}, \tilde{y}]$ is the commutator of vector fields $\tilde{x}$ and $\tilde{y}$. The Nijenhuis tensor does not depend on a choice of vector fields $\tilde{u}$ and $\tilde{v}$ extending the tangent vectors $u$ and $v$ in the point $P$. Obviously the Nijenhuis tensor is alternating and has the following entries (in a local map $x^{1}, \ldots, x^{n}$ ):

$$
N_{j k}^{i}=\frac{\partial A_{k}^{i}}{\partial x^{\alpha}} A_{j}^{\alpha}-\frac{\partial A_{j}^{i}}{\partial x^{\alpha}} A_{k}^{\alpha}+\frac{\partial A_{j}^{\alpha}}{\partial x^{k}} A_{\alpha}^{i}-\frac{\partial A_{k}^{\alpha}}{\partial x^{j}} A_{\alpha}^{i} .
$$


The Haantjes tensor $H(u, v)$ [6] is defined by the formula

$$
H(u, v)=A^{2} N(u, v)+N(A u, A v)-A(N(A u, v)+N(u, A v))
$$

and also is alternating. Haantjes tensor has the following entries:

$$
H_{j k}^{i}=A_{\alpha}^{i} A_{\beta}^{\alpha} N_{j k}^{\beta}+N_{\alpha \beta}^{i} A_{j}^{\alpha} A_{k}^{\beta}-A_{\alpha}^{i} N_{\beta k}^{\alpha} A_{j}^{\beta}-A_{\alpha}^{i} N_{j \beta}^{\alpha} A_{k}^{\beta},
$$

where one must substitute formulae (2.2).

The Nijenhuis and Haantjes tensors define the alternating products in the tangent space which lead to structures of non-associative algebras in the tangent space. Thus in the tangent bundle $T\left(M^{n}\right)$ we have a deformation of structures of non-associative and alternating algebras.

II. We define two linear operators $N_{u}$ and $H_{u}$ depending on vector $u$ which are analogues of the operator $a d_{u}$ for the Lie algebras:

$$
N_{u}(v)=N(u, v), \quad H_{u}(v)=H(u, v) .
$$

These two operators in view of formula (2.3) are connected by the relation

$$
H_{u}=\left[N_{A u}-A N_{u}, A\right] \text {. }
$$

From (2.6) we get

$$
\operatorname{Tr} H_{u}=0, \quad \operatorname{Tr}\left(H_{u} A^{k}\right)=0
$$

for all integers $k$.

We define two symmetric scalar products $(u, v)_{N}$ and $(u, v)_{H}$ which are analogues of the Cartan-Killing forms for the Lie algebras:

$$
(u, v)_{N}=\operatorname{Tr}\left(N_{u} N_{v}\right), \quad(u, v)_{H}=\operatorname{Tr}\left(H_{u} H_{v}\right) .
$$

III.

Proposition 1. If two $(1,1)$ tensor fields $\tilde{A}_{j}^{i}$ and $A_{j}^{i}$ are connected by the relation

$$
\tilde{A}_{j}^{i}(x)=f(x) A_{j}^{i}(x)+g(x) \delta_{j}^{i},
$$

where $f(x)$ and $g(x)$ are arbitrary functions on the manifold $M^{n}$ then the corresponding Haantjes tensors and scalar products $(u, v)_{H}$ are connected by the formulae

$$
\begin{aligned}
\tilde{H}(u, v) & =f^{4}(x) H(u, v), \\
(u, v)_{\tilde{H}} & =f^{8}(x)(u, v)_{H} .
\end{aligned}
$$

Proof. Let us first assume that $\tilde{A}(x)=f(x) A(x)$. From the definition of the Nijenhuis tensor (2.1) we have

$$
\tilde{N}_{u}(v)=f^{2} N_{u}(v)+f(A u)(f) A v-f u(f) A^{2} v-f(A v)(f) A u+f v(f) A^{2} u .
$$

Hence we get

$\tilde{M}_{u}=\tilde{N}_{\tilde{A} u}-\tilde{A} \tilde{N}_{u}=f^{3}\left(N_{A u}-A N_{u}\right)+f^{2}\left(A^{2} u\right)(f) A-2 f^{2}(A u)(f) A^{2}+f^{2} u(f) A^{3}$.

This expression leads to the equality 


$$
\tilde{H}_{u}=\left[\tilde{M}_{u}, \tilde{A}\right]=f^{4}\left[N_{A u}-A N_{u}, A\right]=f^{4} H_{u},
$$

that proves (2.10) in the case $\tilde{A}=f A$.

Let us suppose now that $\tilde{A}=A+g 1$. From (2.7) we get

$$
\tilde{N}_{u}(v)=N_{u}(v)+(A u)(g) v-u(g) A v-(A v)(g) u+v(g) A u .
$$

Hence we obtain

$$
\tilde{M}_{u}=\tilde{N}_{\tilde{A} u}-\tilde{A} \tilde{N}_{u}=N_{A u}-A N_{u}+\left(A^{2} u\right)(g) 1-2(A u)(g) A+u(g) A^{2} .
$$

Thus the equality follows

$$
\tilde{H}_{u}=\left[\tilde{M}_{u}, \tilde{A}\right]=\left[N_{A u}-A N_{u}, A\right]=H_{u} .
$$

Therefore the formula (2.10) is proven for the general case (2.9).

The formula (2.11) follows from (2.10) and the definition of the scalar product $(u, v)_{H}=\operatorname{Tr}\left(H_{u} H_{v}\right)$.

The derived formula (2.10) means that the Haantjes tensor $H(u, v)$ in the contrast with the Nijenhuis tensor $N(u, v)$ possesses the gauge invariance (2.10) under the transformations $(2.9)$ of the $(1,1)$ tensor field $A_{j}^{i}(x)$.

$I V$. Let $e_{k}(x)$ and $e_{s}(x)$ are smooth vector fields of eigenvectors of the operator $A_{j}^{i}(x)$, corresponding to the eigenvalues $\lambda_{k}(x)$ and $\lambda_{s}(x)$. The known Nijenhuis formula [5] follows straightforward from the definition (2.1),

$$
N\left(e_{k}, e_{s}\right)=\left(A-\lambda_{k}\right)\left(A-\lambda_{s}\right)\left[e_{k}, e_{s}\right]+\left(\lambda_{k}-\lambda_{s}\right)\left(e_{k}\left(\lambda_{s}\right) e_{s}+e_{s}\left(\lambda_{k}\right) e_{k}\right) .
$$

The formulae (2.3) and (2.4) imply

$$
H\left(e_{k}, e_{s}\right)=\left(A-\lambda_{k}\right)^{2}\left(A-\lambda_{s}\right)^{2}\left[e_{k}, e_{s}\right] .
$$

For the bilinear tensor

$$
M(u, v)=N(A u, v)-A N(u, v),
$$

we obtain from (2.14)

$$
M\left(e_{k}, e_{s}\right)=-\left(A-\lambda_{k}\right)^{2}\left(A-\lambda_{s}\right)\left[e_{k}, e_{s}\right]+\left(\lambda_{k}-\lambda_{s}\right)^{2} e_{k}\left(\lambda_{s}\right) e_{s} .
$$

The gauge invariance of the Haantjes tensor easily follows from the formula (2.15) for a $(1,1)$ tensor field $A_{j}^{i}(x)$ having $n$ real and distinct eigenvalues. Indeed, operator $\tilde{A}_{j}^{i}(x)(2.9)$ has the same eigenvectors $e_{1}(x), \ldots, e_{n}(x)$ with the eigenvalues

$$
\tilde{\lambda}_{k}(x)=f(x) \lambda_{k}(x)+g(x) .
$$

Therefore we obtain from the formula (2.15)

$$
\tilde{H}\left(e_{k}, e_{s}\right)=\left(\tilde{A}-\tilde{\lambda}_{k}\right)^{2}\left(\tilde{A}-\tilde{\lambda}_{s}\right)^{2}\left[e_{k}, e_{s}\right]=f^{4}(x) H\left(e_{k}, e_{s}\right) .
$$

For the general case of complex eigenvalues and non-diagonal Jordan normal form of $A_{j}^{i}(x)$ one needs the direct proof given above.

$V$. We denote $N_{B}(u, v)$ and $H_{B}(u, v)$ the Nijenhuis and Haantjes tensors determined by an operator tensor field $B_{j}^{i}(x)$. Let 


$$
R=R(A, \lambda(x))=(\lambda(x)-A)^{-1}=\sum_{k=0}^{\infty} \frac{A^{k}}{\lambda^{k+1}}
$$

be the resolvent operator for an operator $A_{j}^{i}(x)$.

Proposition 2. The relations hold

$$
\begin{aligned}
N_{A^{-1}}(u, v) & =A^{-2} N_{A}\left(A^{-1} u, A^{-1} v\right), \\
M_{A^{-1}}(u, v) & =-A^{-3} M_{A}\left(A^{-2} u, A^{-1} v\right), \\
H_{A^{-1}}(u, v) & =A^{-4} H_{A}\left(A^{-2} u, A^{-2} v\right), \\
H_{R}(u, v) & =R^{4} H_{A}\left(R^{2} u, R^{2} v\right) .
\end{aligned}
$$

Proof. Let us first prove (2.19)-(2.21) for an operator tensor field $A(x)$ having real and distinct eigenvalues $\lambda_{1}, \ldots, \lambda_{n}$. The inverse operator $A^{-1}$ has the same eigenvectors $e_{1}, \ldots, e_{n}$ and eigenvalues $\lambda_{1}^{-1}, \ldots, \lambda_{n}^{-1}$. From the Nijenhuis formula (2.14) we obtain

$$
\begin{aligned}
N_{A^{-1}}\left(e_{k}, e_{s}\right)= & \left(A^{-1}-\lambda_{k}^{-1}\right)\left(A^{-1}-\lambda_{s}^{-1}\right)\left[e_{k}, e_{s}\right] \\
& +\left(\lambda_{k}^{-1}-\lambda_{s}^{-1}\right)\left(e_{k}\left(\lambda_{s}^{-1}\right) e_{s}+e_{s}\left(\lambda_{k}^{-1}\right) e_{k}\right) \\
= & A^{2} \lambda_{k}^{-1} \lambda_{s}^{-1}\left(A-\lambda_{k}\right)\left(A-\lambda_{s}\right)\left[e_{k}, e_{s}\right] \\
& +\left(\lambda_{k}-\lambda_{s}\right)\left(\lambda_{k}^{-1} e_{k}\left(\lambda_{s}\right) \lambda_{s}^{-3} e_{s}+\lambda_{s}^{-1} e_{s}\left(\lambda_{k}\right) \lambda_{k}^{-3} e_{k}\right) \\
= & A^{-2} N_{A}\left(A^{-1} e_{k}, A^{-1} e_{s}\right) .
\end{aligned}
$$

Formula (2.19) follows from (2.23) in view of the bilinearity of the Nijenhuis tensor $N(u, v)$. Formula (2.19) was derived by an indirect method in [5].

Formula (2.17) leads to the equality

$$
\begin{aligned}
M_{A^{-1}}\left(e_{k}, e_{s}\right)= & -\left(A^{-1}-\lambda_{k}^{-1}\right)^{2}\left(A^{-1}-\lambda_{s}^{-1}\right)\left[e_{k}, e_{s}\right] \\
& +\left(\lambda_{k}^{-1}-\lambda_{s}^{-1}\right)^{2} e_{k}\left(\lambda_{s}^{-1}\right) e_{s} \\
= & A^{-3} \lambda_{k}^{-2} \lambda_{s}^{-1}\left(A-\lambda_{k}\right)^{2}\left(A-\lambda_{s}\right)\left[e_{k}, e_{s}\right] \\
& -\left(\lambda_{k}-\lambda_{s}\right)^{2} \lambda_{k}^{-2} e_{k}\left(\lambda_{s}\right) \lambda_{s}^{-1} e_{s} \\
= & -A^{-3} M_{A}\left(A^{-2} e_{k}, A^{-1} e_{s}\right)
\end{aligned}
$$

that implies the formula (2.20).

From the formula (2.15) we get

$$
\begin{aligned}
H_{A^{-1}}\left(e_{k}, e_{s}\right) & =\left(A^{-1}-\lambda_{k}^{-1}\right)^{2}\left(A^{-1}-\lambda_{s}^{-1}\right)^{2}\left[e_{k}, e_{s}\right] \\
& =A^{-4} \lambda_{k}^{-2} \lambda_{s}^{-2}\left(A-\lambda_{k}\right)^{2}\left(A-\lambda_{s}\right)^{2}\left[e_{k}, e_{s}\right] \\
& =A^{-4} H_{A}\left(A^{-2} e_{k}, A^{-2} e_{s}\right),
\end{aligned}
$$

and hence formula (2.21) follows.

Operators $A(x)$ with distinct and real eigenvalues form a domain $\mathcal{O}$ in the space of all linear operators. The expressions (2.19)-(2.21) analytically depend on the entries $A_{j}^{i}(x)$ and their derivatives. Therefore the validity of the formulae 
(2.19) $-(2.21)$ in the entire domain $\mathcal{O}$ of the operators $A(x)$ implies their validity for arbitrary operators $A(x)$.

Formula (2.22) follows from (2.21) and from the gauge invariance (2.10) of the Haantjes tensor

$$
H_{\lambda(x)-A}(u, v)=H_{A}(u, v)
$$

for any smooth function $\lambda(x)$.

Remark 1. From (2.22) we obtain that the Haantjes tensor for the resolvent operator $R(A, \lambda(x))$ (2.18) depends analytically on the function $\lambda(x)$ and does not depend on its derivatives. Formula (2.22) after the substitution of the power series (2.18) leads to an infinite family of identities connected with the Haantjes tensor $H_{A}(u, v)$.

\section{Jacobi Tensors and Lie Algebra Structures}

I. We define the following alternating functions of pairs of tangent vectors $u$ and $v \in T_{x}\left(M^{n}\right)$, values of which are linear operators on the tangent space $T_{x}\left(M^{n}\right)$ :

$$
\begin{aligned}
& J_{N}(u, v)=N_{N(u, v)}-\left[N_{u}, N_{v}\right], \\
& J_{H}(u, v)=H_{H(u, v)}-\left[H_{u}, H_{v}\right] .
\end{aligned}
$$

Obviously $J_{N}$ and $J_{H}$ form $(1,3)$ tensors

$$
J_{N}(u, v) w=J_{N i j k}^{s} u^{i} v^{j} w^{k}, \quad J_{H}(u, v) w=J_{H i j k}^{s} u^{i} v^{j} w^{k} .
$$

Tensor $J_{H}$ is gauge invariant as well as the Haantjes tensor, see (2.10). After any transformation (2.9) one gets

$$
\tilde{J}_{H}(u, v)=f^{8}(x) J_{H}(u, v) .
$$

Formulae (3.1) and (3.2) are similar to the definition of the Riemann tensor in the Riemannian geometry

$$
R(u, v)=\nabla_{[u, v]}-\left[\nabla_{u}, \nabla_{v}\right]
$$

The tensors $J_{N}$ and $J_{H}$ have another meaning: they characterize the deviation of the algebraic structures defined by the tensors $N$ and $H$ from the Lie algebra structures. Indeed, from (3.1) and (3.2) we get

$$
\begin{aligned}
& J_{N}(u, v) w=N(N(u, v), w)+N(N(v, w), u)+N(N(w, u), v), \\
& J_{H}(u, v) w=H(H(u, v), w)+H(H(v, w), u)+H(H(w, u), v) .
\end{aligned}
$$

Thus if tensor $J_{N} \equiv 0$ then (3.6) is the Jacobi identity and hence tensor $N(u, v)$ defines the Lie algebra structure in the tangent space. Therefore we name tensors $J_{N}$ and $J_{H}$ the Jacobi tensors. These tensors have the following symmetries:

$$
\begin{gathered}
J(u, v) w=-J(v, u) w, \\
J(u, v) w=J(v, w) u=J(w, u) v .
\end{gathered}
$$

In the index form equality (3.7) implies

$$
J_{H i j k}^{s}=H_{i j}^{\alpha} H_{\alpha k}^{s}+H_{j k}^{\alpha} H_{\alpha i}^{s}+H_{k i}^{\alpha} H_{\alpha j}^{s} .
$$


Symmetries (3.8) and (3.9) lead to the general equality

$$
J_{H i j k}^{s}=\operatorname{sign}(\sigma) J_{H \sigma(i) \sigma(j) \sigma(k)}^{s},
$$

where $\sigma$ is an arbitrary permutation of the indices $i, j$ and $k$ (the same for $J_{N}$ ).

From the identity $\operatorname{Tr} H_{u}=0$ and the formulae (3.2) and (3.11) we obtain that all contractions of the Jacobi tensor $J_{H}$ vanish:

$$
J_{H i j \alpha}^{\alpha}=J_{H i \alpha j}^{\alpha}=J_{H \alpha i j}^{\alpha}=0 .
$$

Contractions of the Jacobi tensor $J_{N i j k}^{s}$ define the differential 2-form $\alpha_{N 0}(u, v)$

$$
J_{N i j \alpha}^{\alpha}=-J_{N i \alpha j}^{\alpha}=J_{N \alpha i j}^{\alpha}=\alpha_{N 0 i j}
$$

II. For a $(1,1)$ tensor field $A_{j}^{i}$ on a manifold $M^{n}$ of small dimension we prove the following facts.

Proposition 3. For $n=2$ the Jacobi tensor vanishes $J_{N}=0$ and the Haantjes tensor $H=0$ (hence $J_{H}=0$ ). For $n=3$ the Jacobi tensor $J_{H}=0$ and the Jacobi tensor $J_{N}=0$ if the differential form $\alpha_{N 0}(u, v)=0$.

Proof. For $n=2$ the equalities $J_{H i j k}^{s}=0$ follow from the symmetry properties (3.11) as well as equalities $H_{i j}^{k}=0$ follow from $H_{i j}^{k}=-H_{j i}^{k}$ and $H_{i \alpha}^{\alpha}=0$.

For $n=3$ the equalities $J_{H i k j}^{s}=0$ follow from (3.11) and (3.12) and the equalities $J_{N i j k}^{s}=0$ follow analogously from (3.13) if $\alpha_{N 0}(u, v)=0$.

Corollary 1. A manifold $M^{n}$ with a $(1,1)$ tensor field $A_{j}^{i}(x)$ for $n=2,3$ possesses a deformation of Lie algebra structures in the tangent bundle $T\left(M^{n}\right)$ defined for $n=2$ by the Nijenhuis tensor $N_{i j}^{k}$ and for $n=3$ by the Haantjes tensor $H_{i j}^{k}$.

For $n=3$ let $e_{1}(x), e_{2}(x), e_{3}(x)$ be the eigenvectors of the operator $A_{j}^{i}(x)$. From the formula $(2.15)$ we obtain

$$
H\left(e_{1}, e_{2}\right)=h_{3} e_{3}, \quad H\left(e_{2}, e_{3}\right)=h_{1} e_{1}, \quad H\left(e_{3}, e_{1}\right)=h_{2} e_{2} .
$$

These formulae describe, in view of the relations (2.7) $\operatorname{Tr} H_{u}=0$, the canonical form of the commutators of all 3-dimensional Lie algebras, determined by the Haantjes tensors $H(u, v)$.

III. We define two bilinear symmetric tensors

$$
\begin{aligned}
& L_{1}(u, v)=N(A u, v)-N(u, A v), \\
& L_{2}(u, v)=H(A u, v)-H(u, A v) .
\end{aligned}
$$

These tensors define two deformations of structures of commutative but not associative algebras in the tangent bundle $T\left(M^{n}\right)$.

Tensors

$$
\begin{aligned}
& L_{3}(u, v)=N(A u, v)+N(u, A v), \\
& L_{4}(u, v)=H(A u, v)+H(u, A v) .
\end{aligned}
$$

are alternating. 
We define two bilinear tensors

$$
\begin{aligned}
K(u, v) & =N(u, A v)-A N(u, v), \\
M(u, v)=-K(v, u) & =N(A u, v)-A N(u, v) .
\end{aligned}
$$

The following relations hold:

$$
\begin{aligned}
K(A u, v)-A K(u, v) & =H(u, v), \\
M(u, A v)-A M(u, v) & =H(u, v) .
\end{aligned}
$$

Thus for the corresponding linear operators $K_{u}(v)=K(u, v)$ and $M_{u}(v)=M(u, v)$ we obtain

$$
\begin{aligned}
K_{u} & =\left[N_{u}, A\right], \quad K_{A u}-A K_{u}=H_{u}, \\
M_{u} & =N_{A u}-A N_{u}, \quad\left[M_{u}, A\right]=H_{u} .
\end{aligned}
$$

If the Haantjes tensor $H(u, v)=0$ then we get from (3.22) for any polynomial $P(A)$,

$$
K_{P(A) u}=P(A) K_{u} .
$$

Thus if $P(A) u=0$ then $P(A) K_{u}=0$. Also when $H(u, v)=0$ we get from (3.23) that all operators $M_{u}$ commute with the operator $A$.

\section{Differential Forms and Conservation Laws}

I. We define the following differetial 1-forms

$$
\omega_{k}(u)=\operatorname{Tr}\left(A^{k} N_{u}\right), \quad k \geqq 0
$$

and differential 2-forms

$$
\begin{gathered}
\alpha_{N k}(u, v)=\omega_{k}(N(u, v))=\operatorname{Tr}\left(A^{k} N_{N(u, v)}\right), \quad k \geqq 0, \\
\alpha_{H k}(u, v)=\omega_{k}(H(u, v))=\operatorname{Tr}\left(A^{k} N_{H(u, v)}\right), \quad k \geqq 0, \\
\beta_{N k}(u, v)=\operatorname{Tr}\left(A^{k}\left[N_{u}, N_{v}\right]\right), \quad k \geqq 1, \\
\beta_{H k}(u, v)=\operatorname{Tr}\left(A^{k}\left[H_{u}, H_{v}\right]\right), \quad k \geqq 1,
\end{gathered}
$$

It is also useful to define the generating forms depending on an arbitrary parameter $\lambda$, for the countable families of forms $(4.1)-(4.5)$, for example 1-form $w(u, \lambda)$

$$
\omega(u, \lambda)=\operatorname{Tr}\left((\lambda-A)^{-1} N_{u}\right)=\sum_{k=0}^{\infty} \frac{\omega_{k}(u)}{\lambda^{k+1}} .
$$

Remark 2. A necessary criteria for the Nijenhuis or Haantjes tensors to determine a Lie-algebraic structure in the tangent bundle $T\left(M^{n}\right)$ consist of the vanishing of the forms $\alpha_{N k}-\beta_{N k}=0$ (for $N$ ) and $\beta_{H k}=0$ (for $H$ ) for all $k \geqq 0$. Indeed, these vanishings follow from (3.1) and (3.2) if $J_{N}=0$ or $J_{H}=0$. Hence we get in view of Proposition 3 and formulae (2.7) that for $n=3$ differential 2-forms $\beta_{H k}(u, v)=0$ for all $k$ and if the differential 2-form $\alpha_{N 0}(u, v)=0$, then $\alpha_{N k}(u, v)=\beta_{N k}(u, v)$ for all $k$. 
Proposition 4. Differential 1-form $\omega_{k}$ satisfies the relation

$$
\omega_{k}(v)=A v\left(\frac{1}{k+1} \operatorname{Tr} A^{k+1}\right)-v\left(\frac{1}{k+2} \operatorname{Tr} A^{k+2}\right)
$$

for any tangent vector $v$. If the differential form $\omega_{k}$ vanishes then all solutions $u^{i}(t, x)$ of the system (1.1) satisfy the conservation law

$$
\frac{\partial}{\partial t}\left(\frac{1}{k+1} \operatorname{Tr} A^{k+1}\left(u^{1}, \ldots, u^{n}\right)\right)=\frac{\partial}{\partial x}\left(\frac{1}{k+2} \operatorname{Tr} A^{k+2}\left(u^{1}, \ldots, u^{n}\right)\right) .
$$

Proof. Expression (4.7) after the substitution of the formulae (4.1) and (2.2) turns into an analytic relation between the entries of the operator $A_{j}^{i}\left(u^{1}, \ldots, u^{n}\right)$ and their derivatives. Therefore it is sufficient to prove formula (4.7) for the domain of operators $A_{j}^{i}\left(u^{1}, \ldots, u^{n}\right)$ having real and distinct eigenvalues $\lambda_{1}, \ldots, \lambda_{n}$. Let $e_{1}, \ldots, e_{n}$ be the corresponding eigenvectors. From the Nijenhuis formula (2.14) we obtain

$$
\begin{aligned}
\omega_{k}\left(e_{j}\right) & =\operatorname{Tr}\left(A^{k} N_{e j}\right)=\sum_{s=1}^{n}\left(\lambda_{j}-\lambda_{s}\right) e_{j}\left(\lambda_{s}\right) \lambda_{s}^{k} \\
& =A e_{j}\left(\sum_{s=1}^{n} \frac{1}{k+1} \lambda_{s}^{k+1}\right)-e_{j}\left(\sum_{s=1}^{n} \frac{1}{k+2} \lambda_{s}^{k+2}\right) \\
& =A e_{j}\left(\frac{1}{k+1} \operatorname{Tr} A^{k+1}\right)-e_{j}\left(\frac{1}{k+2} \operatorname{Tr} A^{k+2}\right) .
\end{aligned}
$$

Formula (4.7) is a consequence of the formula (4.9) for an arbitrary tangent vector $v=v^{1} e_{1}+\ldots+v^{n} e_{n}$.

We represent the expression (4.8) for an arbitrary solution $u^{i}(t, x)$ of the system (1.1) $u_{t}^{i}=A_{j}^{i}\left(u^{1}, \ldots, u^{n}\right) u_{x}^{j}$ in the form

$$
\begin{aligned}
& \frac{\partial}{\partial u^{i}}\left(\frac{1}{k+1} \operatorname{Tr} A^{k+1}(u)\right) \frac{\partial u^{i}}{\partial t}-\frac{\partial}{\partial u^{i}}\left(\frac{1}{k+2} \operatorname{Tr} A^{k+2}(u)\right) \frac{\partial u^{i}}{\partial x} \\
& =A_{j}^{i} u_{x}^{j} \frac{\partial}{\partial u^{i}}\left(\frac{1}{k+1} \operatorname{Tr} A^{k+1}(u)\right)-u_{x}^{i} \frac{\partial}{\partial u^{i}}\left(\frac{1}{k+2} \operatorname{Tr} A^{k+2}(u)\right) \\
& =\omega_{k}\left(u_{x}\right) .
\end{aligned}
$$

Therefore if the differential form $\omega_{k}$ vanishes then any solution $u^{i}(t, x)$ satisfies the conservation law (4.8). Proposition 4 is proven.

Corollary 2. If all differential forms $\omega_{k}$ vanish (for example when the Nijenhuis tensor $N(u, v)=0)$ then for any solution $u^{j}(t, x)$ of the system (1.1) the eigenvalues $\lambda_{i}(u)$ of the operator $A_{j}^{i}\left(u^{1}, \ldots, u^{n}\right)$ satisfy the equations

$$
\lambda_{i t}=\lambda_{i} \lambda_{i x}
$$

Indeed, in this case we obtain from (4.8),

$$
\sum_{i=1}^{n} \lambda_{i}^{k} \lambda_{i t}=\sum_{i=1}^{n} \lambda_{i}^{k+1} \lambda_{i x}
$$


The validity of these equations for all $k$ implies the validity of Eq. (4.12).

\section{Invariant Polynomials and Fibrations of the Algebraic Submanifolds}

I. We define on the tangent space $T_{x}\left(M^{n}\right)$ two invariant polynomials

$$
\begin{aligned}
& P_{N}(u, \lambda)=\operatorname{det}\left(N_{u}-\lambda\right)=\sum_{k=0}^{n} p_{N k}(u) \lambda^{k}, \\
& P_{H}(u, \lambda)=\operatorname{det}\left(H_{u}-\lambda\right)=\sum_{k=0}^{n} p_{H k}(u) \lambda^{k},
\end{aligned}
$$

which are homogeneous functions of all arguments $u$ and $\lambda$ of degree $n$. Coefficients $p_{N k}(u)$ and $p_{H k}(u)$ are homogeneous polynomials of $u$ of degree $n-k$. From the equalities

$$
N_{u}(u)=0, \quad H_{u}(u)=0, \quad H_{u}=\left[M_{u}, A\right]
$$

we get

$$
\begin{gathered}
p_{N n}(u)=(-1)^{n}, \quad p_{N n-1}(u)=(-1)^{n-1} \omega_{0}(u), \quad p_{N 0}(u)=\operatorname{det} N_{u}=0, \\
p_{H n}(u)=(-1)^{n}, \quad p_{H n-1}(u)=\operatorname{Tr} H_{u}=0, \quad p_{H 0}(u)=\operatorname{det} H_{u}=0 .
\end{gathered}
$$

Let $\lambda_{1}(u), \ldots, \lambda_{n}(u)$ be eigenvalues of an operator $N_{u}$. The equalities hold

$$
\begin{gathered}
p_{N n-2}(u)=(-1)^{n} \sum_{i \neq j}^{n} \lambda_{i} \lambda_{j}, \\
\omega_{0}(u)=\operatorname{Tr} N_{u}=\sum_{i=1}^{n} \lambda_{i},(u, u)_{N}=\operatorname{Tr}\left(N_{u} N_{u}\right)=\sum_{i=1}^{n} \lambda_{i}^{2} .
\end{gathered}
$$

Hence we obtain

$$
p_{N n-2}(u)=\frac{(-1)}{2} \omega_{0}^{2}(u)-\frac{(-1)^{n}}{2}(u, u)_{N},
$$

and analogously

$$
p_{H n-2}(u)=-\frac{(-1)^{n}}{2}(u, u)_{H} \text {. }
$$

Polynomials (5.1), (5.2) for $n=2$ have the form

$$
P_{N}(u, \lambda)=\lambda^{2}-\omega_{0}(u) \lambda, \quad P_{H}(u, \lambda)=\lambda^{2} .
$$

For $n=3$ we get from (5.3)-(5.8),

$$
\begin{gathered}
P_{N}(u, \lambda)=-\lambda^{3}+\omega_{0}(u) \lambda^{2}-\frac{1}{2}\left(\omega_{0}^{2}(u)-(u, u)_{N}\right) \lambda \\
P_{H}(u, \lambda)=-\lambda^{3}+\frac{1}{2}(u, u)_{H} \lambda
\end{gathered}
$$

Operators $N_{u}$ and $H_{u}$ in view of (5.1) and (5.2) satisfy the algebraic equations

$$
P_{N}\left(u, N_{u}\right)=0, \quad P_{H}\left(u, H_{u}\right)=0 .
$$


For $n=3$ these equations have the form

$$
\begin{gathered}
N_{u}\left(N_{u}^{2}-\omega_{0}(u) N_{u}+\frac{1}{2} \omega_{0}^{2}(u)-\frac{1}{2}(u, u)_{N}\right)=0, \\
H_{u}\left(H_{u}^{2}-\frac{1}{2}(u, u)_{H}\right)=0 .
\end{gathered}
$$

II. We define in the tangent space $T_{x}\left(M^{n}\right)$ two algebraic submanifolds $V_{N}$ and $V_{H}$ by the equations

$$
\begin{aligned}
& V_{N}: P_{N}(u, 1)=\operatorname{det}\left(N_{u}-1\right)=0, \\
& V_{H}: P_{H}(u, 1)=\operatorname{det}\left(H_{u}-1\right)=0 .
\end{aligned}
$$

The manifolds $V_{N}$ and $V_{H}$ are affine parts of the projective manifolds $\tilde{V}_{N}$ and $\tilde{V}_{H}$ defined by the homogeneous characteristic equations

$$
\tilde{V}_{N}: \operatorname{det}\left(N_{u}-\lambda\right)=0, \quad \tilde{V}_{H}: \operatorname{det}\left(H_{u}-\lambda\right)=0 .
$$

Thus we have two fibrations of algebraic manifolds $V_{N}$ and $V_{H}$ embedded into the tangent bundle $T\left(M^{n}\right)$ or into its projectivization. Complex forms of these manifolds are embedded into the complexification of the tangent bundle $T\left(M^{n}\right)$.

For $n=2$ manifolds $V_{N}$ are straight lines $\operatorname{Tr} N_{u}=1$ ( $V_{H}$ are empty). For $n=3$ manifolds $V_{N}$ and $V_{H}$ are quadrics:

$$
\begin{gathered}
V_{N}:(u, u)_{N}-\omega_{0}^{2}(u)+2 \omega_{0}(u)=2, \\
V_{H}:(u, u)_{H}=2 .
\end{gathered}
$$

$V_{H}$ is invariant under the reflection $u \rightarrow-u$.

Let $G_{H x}$ be the group of all linear automorphisms of the Haantjes tensor $H$ (in a point $\left.x \in M^{n}\right)$, so $g \in G_{H x}$ if for any $u, v \in T_{x}\left(M^{n}\right)$ we have

$$
H(g u, g v)=g H(u, v) .
$$

Polynomial $P_{H}(u)$ and algebraic manifold $V_{H}$ obviously are invariant under the action of the group of automorphisms $G_{H x}$ in $T_{x}\left(M^{n}\right)$.

The Haantjes tensor $H$ (or $N$ ) is called reducible if all operators $H_{u}$ (or $N_{u}$ ) have an invariant subspace $L_{1} \subset T_{x}\left(M^{n}\right)$. This means that $H\left(T_{x}\left(M^{n}\right), L_{1}\right) \subset L_{1}$. In this case the polynomial $P_{H}(u)$ can be factored $P_{H}(u)=P_{H 1}(u) P_{H 2}(u)$ and the algebraic manifold $V_{H}$ has two components, determined by equations $P_{H 1}(u)=$ 0 and $P_{H 2}(u)=0$.

If there exists filtration of different subspaces

$$
L_{1} \subset L_{2} \subset L_{3} \subset \ldots \subset L_{k}=T_{x}\left(M^{n}\right),
$$

invariant with respect to all operators $H_{u}$, or $H\left(T_{x}\left(M^{n}\right), L_{\alpha}\right) \subset L_{\alpha}$ for all $\alpha=$ $1, \ldots, k$, then polynomial $P_{H}(u)$ can be factored into a product of $k$ factors $P_{H}(u)=P_{H 1}(u)=P_{H 1} P_{H 2} \ldots P_{H k}$ and algebraic manifold $V_{H}$ has $k$ components.

If tensor $H$ (or $N$ ) has nontrivial central subspace $L_{c}$ of dimension $d$, i.e.,

$$
H\left(L_{c}, T_{x}\left(M^{n}\right)\right)=0,
$$

then the polynomial $P_{H}(u, 1)(5.2)$ has degree at most $n-d$.

We call tensor $H$ (or $N$ ) nilpotent, if for any $u$ we have $H_{u}^{k}=0$ for some $k$. Obviously the complexification of the manifold $V_{H}$ is empty and polynomial $P_{H}(u)=(-1)^{n}$ if and only if tensor $H$ is nilpotent. 
We call vector $v \in T_{x}\left(M^{n}\right) N$-nilpotent (analogously $H$-nilpotent) if operator $N_{v}$ is nilpotent, $N_{v}^{n}=0$. The set $Z_{N}$ of all $N$-nilpotent vectors is determined by the system of $n-1$ homogeneous equations

$$
p_{N 1}(u)=0, \ldots, p_{N n-1}(u)=0 .
$$

Indeed, for vectors $u$, satisfying (5.22) we get from (5.12),

$$
P_{N}\left(u, N_{u}\right)=(-1)^{n} N_{u}^{n}=0 .
$$

If polynomial $P_{N}(u, \lambda)$ can be factored into a product of $n$ linear factors

$$
P_{N}(u, \lambda)=(-1)^{n} \lambda \prod_{k=1}^{n-1}\left(\lambda-f_{k}(u)\right)
$$

then the set $Z_{N}$ is a linear subspace

$$
f_{1}(u)=0, \ldots, f_{n-1}(u)=0 .
$$

This is the case for the Benney system, studied in Sect. 9.

If all operators $N_{u}$ (or $H_{u}$ ) have a common eigenvector $e$ then $e$ is $N$-nilpotent and therefore satisfies all Eqs. (5.22). Indeed, from the condition

$$
N_{u} e=N(u, e)=\lambda(u) e
$$

we obtain

$$
N_{e}^{2} u=N_{e}(N(e, u))=-\lambda(u) N(e, e)=0 .
$$

Polynomial $P_{N}(u, \lambda)$ in this case has a linear divisor $\lambda-\lambda(u)$.

If the two operator tensor fields $\tilde{A}_{j}^{i}$ and $A_{j}^{i}$ are connected by the gauge transformation (2.9) then in view of (2.10) their Haantjes operators $H_{u}$ are connected by the equality $\tilde{H}_{u}=f^{4} H_{u}$. Therefore the corresponding polynomials (5.2) are connected by the relation

$$
P_{\tilde{H}}(u, \lambda)=P_{H}\left(f^{4} u, \lambda\right) .
$$

III. Let $(v, w)=g_{\alpha \beta} v^{\alpha} w^{\beta}$ be a bilinear form in the tangent space $T_{x}\left(M^{n}\right)$, which can be indefinite and non-symmetric, but it must be non-degenerate.

Lemma 1. If all operators $H_{u}$ are skew-symmetric with respect to a bilinear form $(v, w)$ :

$$
\left(H_{u} v, w\right)+\left(v, H_{u} w\right)=0,
$$

then the polynomial $P_{H}(u)$ is even and the algebraic manifold $V_{H}$ is invariant under the reflection $u \rightarrow-u$. Eigenvalues of the operator $H_{u}$ are symmetric with respect to zero and its image has an even dimension in a general case.

Proof. Let $e_{1}, \ldots, e_{n}$ be a basis in a tangent space $T_{x}\left(M^{n}\right)$, scalar products $\left(e_{i}, e_{j}\right)=B_{i j}$ and $H_{u}\left(e_{i}\right)=H_{u i k} e_{k}$. Then the expression (5.26) is equivalent to the matrix equation

$$
H_{u} B=-B H_{u}^{t} .
$$

For the polynomial $P_{H}(u, \lambda)$ we have 


$$
\begin{aligned}
P_{H}(u, \lambda) & =\operatorname{det}\left(H_{u}-\lambda\right)=\operatorname{det}\left(B^{-1} H_{u} B-\lambda\right)=\operatorname{det}\left(-H_{u}^{t}-\lambda\right) \\
& =\operatorname{det}\left(H_{(-u)}-\lambda\right)=P_{H}(-u, \lambda) .
\end{aligned}
$$

Thus the polynomials $P_{H}(u, \lambda)$ and $P_{H}(u)$ are even and hence the algebraic manifold $V_{H}$ is symmetric under the reflection and eigenvalues of an operator $H_{u}$ are symmetric with respect to zero. Therefore the image space $H_{u}\left(T_{x}\left(M^{n}\right)\right)$ has even dimension.

Lemma 2. If all operators $H_{u}$ are skew-symmetric (see (5.26)) then the Jacobi tensor $J_{H}(u, v)$ for any $u, v$ satisfies the relations

$$
\begin{gathered}
\left(J_{H}(u, v) x, y\right)+\left(x, J_{H}(u, v) y\right)=0, \\
\left(J_{H}(u, v) x, y\right)=\left(v, J_{H}(x, y) u\right) .
\end{gathered}
$$

Proof. Equality (5.29) follows from (5.26) after the substitution of the formula (3.2). Equality (5.30) follows from (5.29) and properties of symmetry of the Jacobi tensor (3.8) and (3.9).

Remark 3. For the symmetric scalar product $(x, y)$ in (5.26) equalities (5.29) and (5.30) coincide with the classical identities for the Riemann tensor

$$
\begin{gathered}
\langle R(u, v) x, y\rangle+\langle R(u, v) y, x\rangle=0, \\
\langle R(u, v) x, y\rangle=\langle R(x, y) u, v\rangle .
\end{gathered}
$$

Remark 4. We define in the space $T_{x}\left(M^{n}\right)+T_{x}\left(M^{n}\right)$ an algebraic submanifold $W_{H}$ by the equation

$$
W_{H}: Q_{H}(u, v)=\operatorname{det}\left(J_{H}(u, v)-1\right)=0 .
$$

Such a manifold is defined also in the space $L^{d}$ of bi-vectors $\xi=u \wedge v$, having dimension $d=n(n-1) / 2$. If the equalities (5.26)) are valid for any $u$ and $v$, then as in Lemma 1 we get that polynomial $Q_{H}(u, v)$ and the manifold $W_{H}$ for any point $x \in M^{n}$ are invariant with respect to two reflections $u \rightarrow-u$ and $v \rightarrow-v$.

$I V$. Let us suppose that the Haantjes tensor $H$ determines a structure of Lie algebra $\mathscr{A}_{x}$ in the tangent space $T_{x}\left(M^{n}\right)$, and let $G_{x}$ be the corresponding Lie group. In this case the polynomial $P_{\mathscr{A}}(u)$ and the manifold $V_{\mathscr{A}}$ are invariant with respect to all automorphisms of the Lie algebra $\mathscr{A}_{x}$, e.g. under the adjoint representation of its Lie group $G_{x}$. Thus the manifold $V_{\mathscr{A}}$ contains together with any point $u$ all its orbit $\operatorname{Ad}_{G x}(u)$.

If $F: \mathscr{A}_{1} \rightarrow \mathscr{A}_{2}$ is a homomorphism of two Lie algebras, then $F\left(V_{\mathscr{A} 1}\right) \subset V_{\mathscr{A} 2}$.

If Lie algebra $\mathscr{A}_{x}$ is semi-simple then operators $H_{u}=\mathrm{ad}_{u}$ are skew-symmetric with respect to the Cartan-Killing form. Thus in view of Lemma 1 the corresponding polynomial $P_{H}(u)$ is even and the manifold $V_{H}$ is invariant under the reflection $u \rightarrow-u$.

Remark 5. Let $T$ be a linear representation of a Lie algebra $\mathscr{A}$ in a linear space $L$. We define an algebraic manifold $V_{T} \subset \mathscr{A}$ by the equation

$$
V_{T}: P_{T}(u)=\operatorname{det}(T(u)-1)=0 \text {. }
$$


It is easy to show that if two representations $T_{1}$ and $T_{2}$ are equivalent then the corresponding polynomials $P_{H 1}(u)$ and $P_{H 2}(u)$ and the algebraic manifolds $V_{T 1}$ and $V_{T 2}$ coincide. If a representation $T$ is reducible, then the algebraic manifold $V_{T}$ is reducible too.

\section{Necessary Criteria for Existence of a Non-Degenerate Hamiltonian Structure}

I. Let us consider a class of Hamiltonian systems (1.1) corresponding to the Poisson brackets

$$
\left\{F_{1}(u), F_{2}(u)\right\}=\int_{-\infty}^{\infty} \frac{\delta F_{1}(u)}{\delta u^{i}(x)} I^{i j}(u) \frac{\delta F_{2}(u)}{\delta u^{j}(x)} d x,
$$

where a skew-symmetric operator $I=\left(I^{i j}\right)$ has the form

$$
I^{i j}(u)=g^{i j}(u) \frac{d}{d x}+b_{\beta}^{i j} u_{x}^{\beta} .
$$

A connection

$$
\Gamma_{j k}^{i}(u)=-g_{j \alpha}(u) b_{k}^{\alpha i}(u)
$$

naturally arises from (6.2). Skew-symmetricity of the Poisson brackets (6.1) implies [7-9] the symmetry condition for the metric, $g^{i j}(u)=g^{j i}(u)$, and the compatibility condition of the connection (6.3) with the metric $g_{i j}$ :

$$
\frac{\partial g^{i j}}{\partial u^{k}}-b_{k}^{i j}-b_{k}^{j i}=\frac{\partial g^{i j}}{\partial u^{k}}+\Gamma_{\alpha k}^{i} g^{\alpha j}+\Gamma_{\alpha k}^{j} g^{i \alpha}=0 .
$$

In [7-9] it is shown that the Poisson brackets (6.1)-(6.2) with a non-degenerate metric $g^{i j}(u)$ satisfy the Jacobi identity if and only if the torsion and the curvature of the connection (6.3) are zero. That means the metric $g_{i j}(u)$ is flat and therefore the operator $I^{i j}(u)$ in appropriate coordinates $\left(u^{1}, \ldots, u^{n}\right)$ takes the form

$$
I^{i j}(u)=q^{i} \delta_{j}^{i} \frac{d}{d x}
$$

with some constant coefficients $q^{i}$.

A Hamiltonian system (1.1) with a Hamiltonian $f\left(u^{1}, \ldots, u^{n}\right)$ has the form

$$
u_{t}^{i}=I^{i \alpha} \frac{\partial f}{\partial u^{\alpha}}=g^{i \alpha}(u) \frac{\partial^{2} f}{\partial u^{\alpha} \partial u^{\beta}} u_{x}^{\beta}+b_{\beta}^{i \alpha}(u) \frac{\partial f}{\partial u^{\alpha}} u_{x}^{\beta} .
$$

In view of (6.5) any Hamiltonian system (6.6) can be transformed to the canonical form

$$
u_{t}^{i}=q^{i} \frac{\partial^{2} f}{\partial u^{i} \partial u^{\alpha}} u_{x}^{\alpha}
$$

II. Let us consider a general system (6.6) with symmetric connection (6.3)

$$
\Gamma_{j k}^{i}=\Gamma_{k j}^{i} \text {. }
$$

Metric $g_{i j}$ (that is not necessarily flat and is not necessarily compatible with the connection (6.8)) defines the bilinear form 
Necessary Conditions for Existence of Non-Degenerate Hamiltonian Structures

$$
(v, w)=g_{\alpha \beta} v^{\alpha} w^{\beta}, \quad v, w \in T_{x}\left(M^{n}\right) .
$$

System (6.6) is connected with the operator tensor field

$$
A_{\beta}^{i}(u)=g^{i \alpha}(u) \frac{\partial^{2} f}{\partial u^{\alpha} \partial u^{\beta}}+b_{\beta}^{i \alpha}(u) \frac{\partial f}{\partial u^{\alpha}} .
$$

The symmetricity of the operator $A$

$$
(A v, w)=(v, A w)
$$

follows from the condition (6.8). Indeed, (6.11) is equivalent to the equation

$$
\sum_{\alpha, \beta, \gamma} g_{\alpha \gamma} A_{\beta}^{\gamma}\left(v^{\alpha} w^{\beta}-v^{\beta} w^{\alpha}\right)=0 \text {. }
$$

The last one after substituting the formulae (6.10) and (6.3) takes the form

$$
\sum_{\alpha, \beta, \gamma}\left(\frac{\partial^{2} f}{\partial u_{\alpha} \partial u_{\beta}}-\Gamma_{\alpha \beta}^{\gamma} \frac{\partial f}{\partial u^{\gamma}}\right)\left(v^{\alpha} w^{\beta}-v^{\beta} w^{\alpha}\right)=0,
$$

and the statement follows. In particular we obtain that the operator $A(6.10)$ for a Hamiltonian system (6.6) is symmetric with respect to the scalar product (6.9) [9]. III.

Theorem 1. The operators $H_{u}$ (2.5) for the Hamiltonian system (6.6) are skewsymmetric with respect to the bilinear form (6.9):

$$
\left(H_{u} v, w\right)+\left(v, H_{u} w\right)=0 .
$$

Proof. The condition (6.14) is invariant. Thus it is enough to check (6.14) for the canonical form (6.7) of the Hamiltonian system. The system (6.7) is a special case of the system of conservation laws $[2,3]$

$$
u_{t}^{i}=f_{x}^{i}=\frac{\partial f^{l}}{\partial u^{\alpha}} u_{x}^{\alpha}
$$

We denote partial derivatives by low indices: $f_{\alpha}^{i}=\partial f^{i} / \partial u^{\alpha}, f_{\alpha \beta}^{l}=\partial^{2} f / \partial u^{\alpha} \partial u^{\beta}$, etc.

System $(6.15)$ is connected with the $(1,1)$ tensor field $A_{\alpha}^{i}(u)=\partial f^{i} / \partial u^{\alpha}$. The corresponding Nijenhuis tensor (2.2) has the following entries:

$$
N_{j k}^{i}=f_{k \alpha}^{i} f_{j}^{\alpha}-f_{j \alpha}^{i} f_{k}^{\alpha} .
$$

A direct calculation of the Haantjes tensor (2.4) for the system of conservation laws (6.15) gives the formula

$$
\begin{aligned}
H_{j k}^{i}= & f_{\alpha}^{i} f_{\beta}^{\alpha} f_{\gamma k}^{\beta} f_{j}^{\gamma}-f_{\alpha}^{i} f_{\beta}^{\alpha} f_{\gamma j}^{\beta} f_{k}^{\gamma} \\
& +f_{\alpha}^{i} f_{\beta j}^{\alpha} f_{\gamma}^{\beta} f_{k}^{\gamma}-f_{\alpha}^{i} f_{\beta k}^{\alpha} f_{\gamma}^{\beta} f_{j}^{\gamma} \\
& +f_{\alpha \beta}^{i} f_{k}^{\alpha} f_{\gamma}^{\beta} f_{j}^{\gamma}-f_{\alpha \beta}^{i} f_{j}^{\alpha} f_{\gamma}^{\beta} f_{k}^{\gamma} .
\end{aligned}
$$

The system (6.7) is embedded into (6.15) where $f^{i}=q^{i} \partial f / \partial u^{i}$. Therefore for the system (6.7) we get from (6.16) 


$$
N_{j k}^{i}=q^{i} q^{\alpha}\left(f_{i k \alpha} f_{\alpha j}-f_{i j \alpha} f_{\alpha k}\right) .
$$

The Haantjes tensor for the system (6.7) in view of (6.17) is determined by the formulae

$$
\begin{gathered}
H_{j k}^{i}=q^{i} \tilde{H}_{j k}^{i}, \\
\tilde{H}_{j k}^{i}=\sum_{\sigma} \operatorname{sign}(\sigma) \sum_{\alpha \beta \gamma}^{n} q^{\alpha} q^{\beta} q^{\gamma} f_{\sigma(i) \alpha} f_{\alpha \beta \sigma(j)} f_{\beta \gamma} f_{\gamma \sigma(k)},
\end{gathered}
$$

where summation is taken over all permutations $\sigma$ of indices $i, j, k$. Coefficients $\tilde{H}_{j k}^{i}$ are skew-symmetric with respect to the transpositions of any two indices $i, j, k$.

The condition of the skew-symmetricity of the operators $H_{u}(6.14)$ is equivalent to the equations

$$
\sum_{\alpha, \beta, \gamma} g_{\alpha \gamma} H_{j \beta}^{\gamma}\left(v^{\alpha} w^{\beta}+v^{\beta} w^{\alpha}\right)=0
$$

for all $j=1, \ldots, n$. Substituting here the formula (6.19) and $g_{\alpha \gamma}=\left(q^{\alpha}\right)^{-1} \delta_{\alpha}^{\gamma}$ we obtain the equations

$$
\sum_{\alpha, \beta} \tilde{H}_{j \beta}^{\alpha}\left(v^{\alpha} w^{\beta}+v^{\beta} w^{\alpha}\right)=0
$$

These equations hold indentically in view of

$$
\tilde{H}_{j \beta}^{\alpha}=-\tilde{H}_{j \alpha}^{\beta}
$$

Theorem 1 is proven.

$I V$. For the Jacobi tensor $J_{H i j k}^{s}$ we denote $J_{H i j k s}=J_{H i j k}^{\alpha} g_{\alpha s}$.

Corollary 3. The Jacobi tensor $J_{H}$ for the Hamiltonian system (6.6) has the following properties:

$$
\begin{aligned}
J_{H}(u, v) w & =-J_{H}(v, u) w \\
J_{H}(u, v) w & =J_{H}(v, w) u=J_{H}(w, u) v, \\
\left(J_{H}(u, v) x, y\right) & +\left(x, J_{H}(u, v) y\right)=0, \\
\left(J_{H}(u, v) x, y\right) & =\left(J_{H}(x, y) u, v\right),
\end{aligned}
$$

equivalent to the relations

$$
J_{H i j k s}=\operatorname{sign}(\sigma) J_{H \sigma(i) \sigma(j) \sigma(k) \sigma(s)}
$$

for all permutations $\sigma$ of four indices $i, j, k, s$.

Proof. The equalities (6.23) follow from the general symmetries (3.8) and (3.9) of the Jacobi tensor and from Lemma 2 based on Theorem 1 for the Hamiltonian system (6.6). The equivalence of (6.23) and (6.24) is simple to verify.

The Haantjes tensor $H(u, v)$ for $n=3$ for any $(1,1)$-tensor field $A_{j}^{i}(x)$ defines the structure of the Lie algebra in each tangent space $T_{x}\left(M^{3}\right)$, see Corollary 1 . Thus in the whole we have the deformation of the structures of the Lie algebras in the tangent bundle $T\left(M^{3}\right)$. For the Hamiltonian system (6.6) this is obvious because of the vanishing of the Jacobi tensor (3.7) for $n=3$ in view of (6.24). 
Theorem 2. For the Hamiltonian system (6.6) for $n=3$ the following alternative is true:

1) The Haantjes tensor $H(u, v)$ is zero. Then the system (6.6) possesses the Riemann invariants and is integrable by the generalized hodograph transformation.

2) The Haantjes tensor $H(u, v)$ is non-zero. Then the arising Lie algebra for each tangent space $T_{x}\left(M^{3}\right)$ is simple, so it is isomorphic either to so (3) or sl (3). The Cartan-Killing form $(u, v)_{H}=\operatorname{Tr}\left(H_{u} H_{v}\right)$ defines the non-degenerate metric $h_{i j}$ on the manifold $M^{3}$ that has to be conformally flat and therefore has to satisfy the classical Weyl-Schouten equations:

$$
R_{i j k}=R_{i j, k}-R_{i k, j}-\frac{1}{4}\left(h_{i k} R_{, j}-h_{i j} R_{, k}\right)=0 .
$$

Proof. 1) The existence of the Riemann invariants provided that the Haantjes tensor $H(u, v)=0$ is proven in our paper [11]. The integrability by the generalized hodograph transformation follows from the Tsarev theorem [19].

2) The Haantjes tensor (6.19), (6.20) for the Hamiltonian system in the canonical form (6.7) has only the following non-zero components:

$$
H_{12}^{3}=-H_{21}^{3}=q^{3} c(x), \quad H_{23}^{1}=-H_{32}^{1}=q^{1} c(x), \quad H_{31}^{2}=-H_{13}^{2}=q^{2} c(x),
$$

where $c(x)=\tilde{H}_{12}^{3}(x)$ (constants $q^{1}, q^{2}, q^{3}$ are nonzero because the metric $g_{i j}$ is nondegenerate). Therefore the Lie algebra in $T_{x}\left(M^{3}\right)$ is simple if $c(x) \neq 0$ and is commutative if $c(x)=0$.

From (6.26) we get for the metric $(u, v)_{H}$ :

$$
h_{i j}(x)=\sum_{\alpha, \beta}^{3} H_{i \beta}^{\alpha} H_{j \alpha}^{\beta}=\left(-c(x) q^{1} q^{2} q^{3}\right) g_{i j}(x) \text {. }
$$

Thus the metric $h_{i j}(x)$ is conformal to the flat metric $g_{i j}(x)$. That follows also from the fact that the Lie algebra in $T_{x}\left(M^{3}\right)$ is simple and its operators $\operatorname{ad}_{u}=H_{u}$ are skew-symmetric with respect to the two metrics $h_{i j}(x)$ and $g_{i j}(x)$.

Any conformally flat metric $h_{i j}(x)$ satisfies the Weyl-Schouten equations (6.25) [18] where $R_{i j}$ is the Ricci tensor and $R$ is the scalar curvature of the metric $h_{i j}$; the $R_{i j, k}$ and $R, j$ are their covariant derivatives.

Theorem 2 is proven.

Theorem 3. The Haantjes tensor $H(u, v)$ for the Hamiltonian system (6.6) for $n=4$ defines the structure of the Lie algebra $\mathscr{A}_{4}(x)$ in each tangent space $T_{x}\left(M^{4}\right)$. The Cartan-Killing form $h_{i j}=\left(e_{i}, e_{j}\right)_{H}$ is degenerate. If $h_{i j}=0$ then the Haantjes tensor $H_{j k}^{i}=0$. Thus the Lie algebra $\mathscr{A}_{4}(x)$ can be neither simple nor nilpotent.

Proof. The Jacobi tensor $J_{H}$ for a Hamiltonian system (6.6) for $n=4$ has in view of (6.24) only the following nonzero components

$$
J_{H \sigma(1) \sigma(2) \sigma(3) \sigma(4)}=\operatorname{sign}(\sigma) J_{H 1234} .
$$

In the coordinates $u^{1}, u^{2}, u^{3}, u^{4}$, where the system (6.6) has a canonical form (6.7), we have 


$$
J_{H 123}^{4}=\sum_{\alpha=1}^{4}\left(H_{12}^{\alpha} H_{\alpha 3}^{4}+H_{23}^{\alpha} H_{\alpha 1}^{4}+H_{31}^{\alpha} H_{\alpha 2}^{4}\right) .
$$

After the substitution of the formulae (6.19), (6.20) we find that each summand in (6.27) is equal to zero because symbol $\tilde{H}_{j k}^{i}(6.20)$ is equal to zero when any two of the indices $i, j, k$ coincide. Therefore the Jacobi tensor $J_{H}$ vanishes and hence the Haantjes tensor $H$ in view of (3.7) defines the structure of the Lie algebra $\mathscr{A}_{4}(x)$ in each tangent space $T_{x}\left(M^{4}\right)$.

As known, the Cartan-Killing form for any 4-dimensional Lie algebra is degenerate. Thus rank $r$ of the form $h_{i j}=\left(e_{i}, e_{j}\right)_{H}$ has to be $0 \leqq r \leqq 3$.

The Haantjes tensor $H_{j k}^{i}$ in the canonical coordinates (6.7) has the form (6.19), (6.20). For $n=4$ we denote

$$
\tilde{H}_{23}^{4}=c_{1}, \quad \tilde{H}_{34}^{1}=c_{2}, \quad \tilde{H}_{41}^{2}=c_{3}, \quad \tilde{H}_{12}^{3}=c_{4} .
$$

All entries $H_{j k}^{i}$ are the linear combinations of $c_{1}, c_{2}, c_{3}, c_{4}$. Substituting these expressions and (6.19) into the formula

$$
h_{i j}=\left(e_{i}, e_{j}\right)_{H}=\sum_{\alpha, \beta}^{3} H_{i \alpha}^{\beta} H_{j \beta}^{\alpha},
$$

we obtain the relations

$$
h_{i j}=-2 \frac{c_{i} c_{j}}{q_{i} q_{j}}, \quad h_{i i}=-\frac{2 q_{i}}{q}\left(q_{j} c_{j}^{2}+q_{k} c_{k}^{2}+q_{s} c_{s}^{2}\right),
$$

where

$$
q=q_{1} q_{2} q_{3} q_{4}, \quad i \neq j \neq k \neq s .
$$

Hence it follows that if the metric $h_{i j}=0$, then all $c_{k}=0$, and therefore the Haantjes tensor $H_{j k}^{i}=0$. Theorem 3 is proven.

Remark 6. If the Haantjes tensor $H(v, w)$ defines the structure of the simple Lie algebra in each tangent space $T_{x}\left(M^{n}\right)$ then the bilinear form (6.9) is proportional in view of (6.14) to the Cartan-Killing form $(v, w)_{H}=\operatorname{Tr}\left(H_{v} H_{w}\right)$. Therefore the metric $h_{i j}(x)$ defined on the manifold $M^{n}$ by the form $(v, w)_{H}$ must be conformally flat, or the Weyl tensor of the conformal curvature $C_{j k e}^{i}(x)$ for the metric $h_{i j}(x)$ for $n>3$ must be zero (for $n=3$ this tensor is zero identically [18]).

$V$. The properties of a Hamiltonian system (6.6) for $n=3,4$ described by Theorems 2 and 3 do not depend on the metric $g_{i j}$ and are the properties of the Haantjes tensor only. Therefore these properties are the definite necessary conditions for the general system (1.1) for $n=3,4$ to be Hamiltonian. In the following Theorem 4 we describe invariant necessary conditions for the existence of a Hamiltonian structure for a system (1.1) for all $n$.

Theorem 4. If a system (1.1) possesses some Hamiltonian structure (6.6) then the following necessary conditions are fulfilled in any point $x \in M^{n}$ and for any vectors $u, v, w \in T_{x}\left(M^{n}\right)$ :

1) The polynomial $P_{H}(u)=\operatorname{det}\left(H_{u}-1\right)$ is even.

2) The algebraic manifold $V_{H} \subset T_{x}\left(M^{n}\right)$ is invariant under the involution $u \rightarrow-u$. 
3) The eigenvalues of an operator $H_{u}$ are symmetric with respect to zero and the dimension of its image $H_{u}\left(T_{x}\left(M^{n}\right)\right)$ is even in a general case.

4) The function $\omega(u, v, w)=\operatorname{Tr}\left(H_{u} H_{v} H_{w}\right)$ is skew-symmetric with respect to the transpositions of $u, v, w$.

5) The differential 2-forms $\beta_{H k}(u, v)=\operatorname{Tr}\left(A^{k}\left[H_{u}, H_{v}\right]\right)=0$.

6) The following transvections are equal to zero

$$
\begin{gathered}
J_{H i j \beta}^{\alpha}\left(A^{m}\right)_{\alpha}^{\beta}=\operatorname{Tr}\left(J_{H i j} A^{m}\right)=0, \\
\operatorname{Tr}\left(H_{u}^{k} H_{v}^{m}\right)=0, \quad k+m=2 s+1 .
\end{gathered}
$$

Proof. The conditions 1), 2) and 3) follow from Lemma 1 in view of Theorem 1. Condition 4) is equivalent to the equality

$$
\operatorname{Tr}\left(H_{u}\left(H_{v} H_{w}+H_{w} H_{v}\right)\right)=0 .
$$

The proof of the equality (6.28) and the conditions 4), 5) and 6) is based on the following lemma known in the linear algebra.

Lemma 3. If an operator $A$ is symmetric and an operator $B$ is skew-symmetric with respect to a non-degenerate bilinear form $(v, w)=g_{\alpha \beta} v^{\alpha} w^{\beta}$ :

$$
(A v, w)=(v, A w), \quad(B v, w)=-(v, B w),
$$

then $\operatorname{Tr}(A B)=0$.

Indeed, from (6.29) one gets

$$
(A B v, w)=-(v, B A w) .
$$

This equality implies

$$
A B g=-g(B A)^{t}, \quad g^{-1} A B g=-(B A)^{t} .
$$

Hence

$$
\operatorname{Tr}(A B)=\operatorname{Tr}\left(g^{-1} A B g\right)=\operatorname{Tr}\left(-(B A)^{t}\right)=-\operatorname{Tr}(A B),
$$

and the claim follows.

If a system (1.1) is Hamiltonian and so has the form (6.6) then the corresponding operator $A_{j}^{i}(x)$ is symmetric with respect to the bilinear form (6.9), see (6.11). Operators $H_{u}$ are skew-symmetric in view of Theorem 1, see (6.14). Operators $J_{H}(u, v)$ are skew-symmetric in view of the Corollary 3, see (6.23). Therefore the conditions 4), 5),6) and the equalities (6.28) follow from Lemma 3.

Theorem 4 is proven.

Remark 7. It is easy to derive from Lemma 3 the vanishing of many other transvections, for example

$$
\begin{aligned}
\operatorname{Tr}\left(J_{H}^{k}(u, v) H_{w}^{m}\right) & =0, & & k+m=2 s+1, \\
\operatorname{Tr}\left(H_{u}^{k}\left[H_{v}^{l}, H_{w}^{m}\right]\right) & =0, & & k+l+m=2 s, \\
\operatorname{Tr}\left(A^{k}\left[H_{u}^{l}, H_{v}^{m}\right]\right) & =0, & & l+m=2 s, \\
\operatorname{Tr}\left(H_{u}^{k}\left(H_{v}^{l} H_{w}^{m}+H_{w}^{m} H_{v}^{l}\right)\right) & =0, & & k+l+m=2 s+1 .
\end{aligned}
$$


These equalities are also the necessary conditions for a system (1.1) to be Hamiltonian.

$V I$. Let us consider the bilinear tensor $M(u, v)(3.23)$.

Theorem 5. Linear operators $M_{u}=N_{A u}-A N_{u}$ for a Hamiltonian system (6.6) are symmetric with respect to the bilinear form (6.9):

$$
\left(M_{u} v, w\right)=\left(v, M_{u} w\right) .
$$

Proof. The condition (6.31) is invariant. Therefore it is enough to prove it for the canonical form (6.7) of a Hamiltonian system (6.6). Tensor $M_{j k}^{i}$ is connected with the Nijenhuis tensor $N_{j k}^{i}$ in view of (3.23) by the formulae

$$
M_{j k}^{i}=N_{\alpha k}^{i} A_{j}^{\alpha}-N_{j k}^{\alpha} A_{\alpha}^{i} .
$$

This expression for a general system of conservation laws (6.15) in view of (6.16) takes the form

$$
M_{j k}^{i}=f_{k \alpha}^{i} f_{\beta}^{\alpha} f_{j}^{\beta}-f_{\alpha \beta}^{i} f_{j}^{\alpha} f_{k}^{\beta}-f_{k \beta}^{\alpha} f_{j}^{\beta} f_{\alpha}^{i}+f_{j \beta}^{\alpha} f_{k}^{\beta} f_{\alpha}^{i} .
$$

Hence we obtain after the substitution $f^{i}=q^{i} \partial f / \partial u^{i}$ for the canonical form (6.7):

$$
\begin{gathered}
M_{j k}^{i}=q^{i} \tilde{M}_{j k}^{i}, \\
\tilde{M}_{j k}^{i}=\sum_{\alpha, \beta} q^{\alpha} q^{\beta}\left(f_{i k \alpha} f_{\alpha \beta} f_{\beta j}-f_{i \alpha \beta} f_{\alpha j} f_{\beta k}-f_{k \alpha \beta} f_{\alpha j} f_{\beta i}+f_{j \alpha \beta} f_{\alpha l} f_{\beta k}\right) .
\end{gathered}
$$

Symmetricity condition (6.31) is equivalent to the equations

$$
g_{\alpha \gamma} M_{j \beta}^{\gamma}=g_{\beta \gamma} M_{j \alpha}^{\gamma}, \quad g M_{j}=M_{j}^{t} g
$$

for all $\alpha, \beta$ and $j=1, \ldots, n$. Substituting here the formulae (6.34) and $g_{\alpha \gamma}=$ $\left(q^{\alpha}\right)^{-1} \delta_{\alpha}^{\gamma}$ we obtain the equations

$$
\tilde{M}_{j k}^{i}=\tilde{M}_{j i}^{k},
$$

which hold obviously for the coefficients (6.35).

Theorem 5 is proven.

Remark 8. The operators $H_{u}$ are the commutators $H_{u}=\left[M_{u}, A\right]$, see (2.6) and (3.23). Therefore the skew-symmetricity of the operators $H_{u}$ (Theorem 1) follows from the symmetricity of the operators $A$ and $M_{u}$ (Theorem 5).

Corollary 4. If metric $g_{i j}$ for a Hamiltonian system (6.6) is positively (or negatively) defined then the scalar product

$$
(u, v)_{M}=\operatorname{Tr}\left(M_{u} M_{v}\right)
$$

is non-negatively defined and the scalar product

$$
(u, v)_{H}=\operatorname{Tr}\left(H_{u} H_{v}\right)
$$


is non-positively defined.

Indeed, for the canonical form (6.7) we have in view of (6.34) and (6.37),

$$
(u, u)_{M}=\sum_{\alpha \beta} q^{\alpha} q^{\beta} \tilde{M}_{u \beta}^{\alpha} \tilde{M}_{u \alpha}^{\beta}=\sum_{\alpha, \beta}^{n} q^{\alpha} q^{\beta}\left(\tilde{M}_{u \beta}^{\alpha}\right)^{2} \geqq 0,
$$

because $q^{\alpha} q^{\beta}>0$. Analogously in view of (6.19) and (6.22) we get

$$
(u, u)_{H}=\sum_{\alpha, \beta} q^{\alpha} q^{\beta} \tilde{H}_{u \beta}^{\alpha} \tilde{H}_{u \alpha}^{\beta}=-\sum_{\alpha, \beta} q^{\alpha} q^{\beta}\left(\tilde{H}_{u \beta}^{\alpha}\right)^{2} \leqq 0 .
$$

Theorem 6. If a system (1.1) possesses some Hamiltonian structure (6.6) then the following necessary conditions are satisfied in any point $x \in M^{n}$ and for any vectors $u, v, w$ in the tangent space $T_{x}\left(M^{n}\right)$ :

1) The polynomials

$$
\begin{aligned}
P_{k}(u) & =\operatorname{det}\left(\left[M_{u}, A^{k}\right]-1\right), \\
Q(u, v) & =\operatorname{det}\left(\left[M_{u}, M_{v}\right]-1\right), \\
R(u, v) & =\operatorname{det}\left(\left[H_{u}, H_{v}\right]-1\right),
\end{aligned}
$$

and the corresponding algebraic manifolds are invariant with respect to two involutions $u \rightarrow-u$ and $v \rightarrow-v$.

2) The eigenvalues of the operators

$$
\left[M_{u}, A^{k}\right], \quad\left[M_{u}, M_{v}\right], \quad\left\{H_{u}, M_{v}\right\}=H_{u} M_{v}+M_{v} H_{u}
$$

are symmetric with respect to zero.

3) The differential 2-forms vanish

$$
\gamma_{k}=\operatorname{Tr}\left(A^{k}\left[M_{u}, M_{v}\right]\right)=0 .
$$

The proof of these necessary conditions is based on the fact that operators $(6.40)$ are skew-symmetric with respect to the bilinear form (6.9) and on Lemma 1.

A wide variety of necessary conditions for a system (1.1) to be Hamiltonian can be derived analogously to (6.30) from the fact that the operators $A$ and $M_{u}$ are symmetric and the operators $H_{u}$ are skew-symmetric with respect to the bilinear form (6.9).

$V I I$. The properties of symmetry of the Nijenhuis tensor with respect to the bilinear form (6.9) are described by the following theorem.

Theorem 7. The Nijenhuis tensor $N$ for a Hamiltonian system (6.6) satisfies the three identities

$$
\begin{gathered}
(N(u, v), w)+(N(v, w), u)+(N(w, u), v)=0, \\
(A N(u, v), w)+(A N(v, w), u)+(A N(w, u), v)=0, \\
\left(A^{2} N(u, v), w\right)+\left(A^{2} N(v, w), u\right)+\left(A^{2} N(w, u), v\right)=(H(u, v), w),
\end{gathered}
$$

for any vectors $u, v, w \in T_{x}\left(M^{n}\right)$. 
Proof. These identities are invariant. So it is enough to prove them in the coordinates where metric $g_{i j}$ is diagonal and system (6.6) has the canonical form (6.7). The identities (6.42)-(6.44) are equivalent to the following tensor relations:

$$
\begin{gathered}
N_{i j}^{\alpha} g_{\alpha k}+N_{j k}^{\alpha} g_{\alpha i}+N_{k i}^{\alpha} g_{\alpha j}=0, \\
N_{i j}^{\alpha} A_{\alpha}^{\beta} g_{\beta k}+N_{j k}^{\alpha} A_{\alpha}^{\beta} g_{\beta i}+N_{k i}^{\alpha} A_{\alpha}^{\beta} g_{\beta j}=0, \\
N_{i j}^{\alpha} A_{\alpha}^{\gamma} A_{\gamma}^{\beta} g_{\beta k}+N_{j k}^{\alpha} A_{\alpha}^{\gamma} A_{\gamma}^{\beta} g_{\beta i}+N_{k i}^{\alpha} A_{\alpha}^{\gamma} A_{\gamma}^{\beta} g_{\beta j}=H_{i j}^{\alpha} g_{\alpha k} .
\end{gathered}
$$

These relations are valid identically after the substitution of the formulae (6.18) -(6.20) and $A_{\alpha}^{i}=q^{i} f_{i \alpha}$ for the canonical form (6.7).

The identity (6.44) can also be derived in a pure algebraic way as a consequence of the two identities (6.42) and (6.43), the relation (2.4) connecting the Haantjes and the Nijenhuis tensors and the symmetricity relation (6.11) for the operator $A$. Theorem 7 is proven.

Remark 9. The property of the symmetricity of the operators $M_{u}=N_{A u}-A N_{u}$ (6.31) follows from the identities (6.42) and (6.43). Indeed, substituting into (6.42) $A u$ instead of $u$ and subtracting from the obtained expression the identity (6.43) we get in view of (6.11)

$$
\left(\left(N_{A u}-A N_{u}\right) v, w\right)-\left(\left(N_{A u}-A N_{u}\right) w, v\right)=0,
$$

that coincides with (6.31). This relation for the tensor $M(u, v)(3.19)$ takes the form

$$
(M(u, v), w)=(M(u, w), v) .
$$

The Haantjes tensor for a Hamiltonian system (6.6) satisfies in view of the formulae (6.19), (6.20) the identities

$$
(H(u, v), w)=(H(v, w), u)=(H(w, u), v) .
$$

These relations are also the immediate consequence of the identity (6.44) along with the skew-symmetricity of the operators $H_{u}$.

Remark 10. The three identities (6.42)-(6.44) are equivalent to the general identity

$$
(P(A) N(u, v), w)+(P(A) N(v, w), u)+(P(A) N(w, u), v)=(H(u, v), w),
$$

where $P(A)$ is the quadratic polynomial

$$
P(A)=A^{2}+p A+q
$$

with arbitrary coefficients $p$ and $q$.

If two vectors $u$ and $v$ belong to the kernel of the operator $P(A)$, or

$$
P(A) u=0, \quad P(A) v=0,
$$

then the identity $(6.45)$ yields

$$
H(u, v)=P(A) N(u, v) .
$$

The formula (6.47) under the conditions (6.46) generalizes the Haantjes formula (2.15) for the Hamiltonian system (6.6). 
Remark 11. The Haantjes tensor $H(u, v)$ for the Hamiltonian system (6.6) satisfies the following identity

$$
(T(u, v), w)+(T(v, w), u)+(T(w, u), v)=0,
$$

where the bilinear tensor $T(u, v)$ is

$$
T(u, v)=H(u, P(A) v)+H(Q(A) u, v)-(P(A)+Q(A)) H(u, v),
$$

and $P(A)$ and $Q(A)$ are arbitrary polynomials of $A$ (could be also arbitrary symmetric operators). This identity easily follows from the symmetricity of the operator $A$ and skew-symmetricity of the operators $H_{u}$.

VIII. Let an operator tensor field $A(x)$ have a multiple eigenvalue $\lambda_{k}(x)$ with a multiplicity $n_{k} \geqq 2$. Let $L_{k x} \subset T_{x}\left(M^{n}\right)$ be the field of the corresponding eigenspaces $\left(A-\lambda_{k}\right) L_{k}=0$ and $e(x), g(x) \in L_{k x}$ be arbitrary eigenvector fields.

Theorem 8. If the operator $A(x)$ corresponds to a Hamiltonian system (1.1) then

1) The relations hold

$$
\begin{gathered}
N\left(L_{k}, L_{k}\right) \subset L_{k}, \quad M\left(L_{k}, L_{k}\right)=0, \quad H\left(L_{k}, L_{k}\right)=0, \\
\left(A-\lambda_{k}\right)^{3}[e(x), g(x)]=0 .
\end{gathered}
$$

2) If the Jordan normal form of the operator $A(x)$ is diagonal then the field of eigenspaces $L_{k}$ is integrable and $N\left(L_{k}, L_{k}\right)=0$.

Proof. For the eigenvector fields $e(x), g(x) \in L_{k x}$ we have

$$
M_{e}=N_{A e}-A N_{e}=\left(\lambda_{k}-A\right) N_{e} .
$$

Operators $M_{u}$ for a Hamiltonian system (1.1) are symmetric with respect to the nondegenerate bilinear form (6.9). Thus from (6.11), (6.31) and (6.50) we obtain

$$
\left(M_{e} g, v\right)=\left(g, M_{e} v\right)=\left(g,\left(\lambda_{k}-A\right) N_{e} v\right)=\left(\left(\lambda_{k}-A\right) g, N_{e} v\right)=0,
$$

where $v \in T_{x}\left(M^{n}\right)$ is an arbitrary vector. Equality (6.51) obviously implies

$$
M_{e} g=\left(\lambda_{k}-A\right) N(e, g)=0 .
$$

Hence the relations (6.48) follow.

The equality (6.49) follows from (6.52) after substituting the Nijenhuis formula (2.14) for $\lambda_{k}=\lambda_{s}$ :

$$
N(e(x), g(x))=\left(A-\lambda_{k}\right)^{2}[e(x), g(x)] .
$$

If the Jordan normal form of the operator $A(x)$ is diagonal then from (6.49) we obtain

$$
[e(x), g(x)] \in L_{k x}
$$

and therefore the field of eigenspaces $L_{k x}$ is integrable. From (6.54) and (6.53) we obtain $N(e, g)=0$. Theorem 8 is proven.

$I X$. A vector $w \in T_{x}\left(M^{n}\right)$ is called central vector for the Nijenhuis tensor if the operator $N_{w}=0$, or $N(w, u)=0$ for all $u$. Linear subspace of all central vectors is called the central subspace $Z$. 
Proposition 5. If the Nijenhuis tensor $N(u, v)$ for a Hamiltonian system (6.6) has a central subspace $Z$ of a dimension $k$ then all vectors $N(u, v)$ belong to $a$ proper subspace $L$ of the dimension $n-k$, the image $A N(u, v)$ belongs to the same subspace $L$.

Proof. Let $L$ be a subspace orthogonal to the central subspace Z. Obviously $L$ has dimension $n-k$. For any central vector $w \in Z$ the identities (6.42) and (6.43) imply

$$
(N(u, v), w)=0, \quad(A N(u, v), w)=0 .
$$

Hence we obtain

$$
N(u, v) \in L, \quad A N(u, v) \in L .
$$

\section{Necessary Criteria for the Existence of Two Non-Degenerate Hamiltonian Structures}

I. Let us suppose that system (1.1) has two Hamiltonian structures (6.6) with two non-degenerate metrics $\left(g_{i j}\right)_{1}$ and $\left(g_{i j}\right)_{2}$. In this case we have the $(1,1)$ tensor field $B_{i}^{j}=g_{1 i \alpha} g_{2}^{\alpha j}$ on the manifold $M^{n}$. Two Hamiltonian structures are in general position if all eigenvalues of the operator $B=g_{1} g_{2}^{-1}$ are distinct (eigenvalues of the operator $B$ can be complex).

Theorem 9. If a system (1.1) has two linear independent non-degenerate Hamiltonian structures then

1) All operators $A, M_{u}$ and $H_{u}$ commute with the operator $B=g_{1} g_{2}^{-1}$.

2) Tensors $M(u, v)$ and $H(u, v)$ are reducible, or all operators $M_{u}$ and $H_{u}$ have a non-trivial invariant subspace. The polynomials defined on the tangent space $T_{x}\left(M^{n}\right)$

$$
\begin{aligned}
& P_{M}(u)=\operatorname{det}\left(M_{u}-\lambda\right), \\
& P_{H}(u)=\operatorname{det}\left(H_{u}-\lambda\right),
\end{aligned}
$$

are reducible.

3) If operator $B$ has $k$ distinct (complex) eigenvalues then polynomials (7.1) and (7.2) have at least $k$ (complex) divisors.

4) If two Hamiltonian structures are in general position then the Haantjes tensor vanishes: $H(u, v) \equiv 0$ and all operators $M_{u}$ and $A$ reduce to the diagonal form simultaneously. The polynomial (7.1) decomposes into a product of $n$ linear factors.

Proof. 1) If a system (1.1) is Hamiltonian with respect to two Hamiltonian structures then in view of Theorem 1 and Theorem 5 operators $A, M_{u}$ and $H_{u}$ satisfy the following identities with respect to the two bilinear forms $(u, v)_{1}$ and $(u, v)_{2}$ :

$$
\begin{gathered}
(A v, w)_{1}=(v, A w)_{1}, \quad(A v, w)_{2}=(v, A w)_{2}, \\
\left(M_{u} v, w\right)_{1}=\left(v, M_{u} w\right)_{1}, \quad\left(M_{u} v, w\right)_{2}=\left(v, M_{u} w\right)_{2}, \\
\left(H_{u}, v, w\right)_{1}=-\left(v, H_{u} w\right)_{1}, \quad\left(H_{u} v, w\right)_{2}=-\left(v, H_{u} w\right)_{2} .
\end{gathered}
$$


Hence we get for the corresponding matrices the equalities:

$$
\begin{array}{cl}
g_{1}^{-1} A g_{1}=A^{t}, & g_{2}^{-1} A g_{2}=A^{t}, \\
g_{1}^{-1} M_{u} g_{1}=M_{u}^{t}, & g_{2}^{-1} M_{u} g_{2}=M_{u}^{t}, \\
g_{1}^{-1} H_{u} g_{1}=-H_{u}^{t}, & g_{2}^{-1} H_{u} g_{2}=-H_{u}^{t} .
\end{array}
$$

These equalities lead to the commutativity relations with $B=g_{1} g_{2}^{-1}$

$$
A B=B A, \quad M_{u} B=B M_{u}, \quad H_{u} B=B H_{u} .
$$

$2)-3)$ In view of (7.4) it is obvious that the reducibility of the tensors $M(u, v)$ and $H(u, v)$ follows from the classical Schur's lemma [17]. Let $P_{B}(\lambda)$ be the characteristic polynomial of the operator $B$

$$
P_{B}(\lambda)=\operatorname{det}(B-\lambda), \quad P_{B}(B)=0 .
$$

Polynomial $P_{B}(\lambda)$ has the form

$$
P_{B}(\lambda)=(-1)^{n} \prod_{i=1}^{k}\left(\lambda-\lambda_{i}\right)^{n_{i}},
$$

where $\lambda_{i}$ are the eigenvalues of the operator $B$ and $n_{i}$ are their multiplicities. Let $L_{i} \subset R^{n}=T_{x}\left(M^{n}\right)$ be a maximal subspace annihilated by the polynomial

$$
p_{i}(B)=\left(B-\lambda_{i}\right)^{n_{i}}, \quad p_{i}(B)\left(L_{i}\right)=0 .
$$

Obviously we have $\operatorname{dim} L_{i}=n_{i}$ and

$$
L_{1}+L_{2}+\ldots+L_{k}=R^{n}, \quad n_{1}+n_{2}+\ldots+n_{k}=n .
$$

Operators $A, M_{u}$ and $H_{u}$ commute with operator $p_{i}(B)(7.7)$ and thus the equalities follow:

$$
p_{i}(B)\left(A\left(L_{l}\right)\right)=0, \quad p_{i}(B)\left(M_{u}\left(L_{i}\right)\right)=0, \quad p_{i}(B)\left(H_{u}\left(L_{i}\right)\right)=0 .
$$

Hence

$$
A\left(L_{i}\right) \subset L_{i}, \quad M_{u}\left(L_{i}\right) \subset L_{i}, \quad H_{u}\left(L_{i}\right) \subset L_{i} .
$$

Therefore all operators $M_{u}, H_{u}$ and $A$ have a block-diagonal structure in the decomposition (7.8). If operator $B$ has $k \geqq 2$ distinct eigenvalues then the operators $M_{u}, H_{u}$ and $A$ have $k$ diagonal blocks in the decomposition (7.8). In this case the polynomials (7.1) and (7.2) are reducible and have $k$ divisors.

Let $k=1$ and thus

$$
P_{B}(\lambda)=(-1)^{n}\left(\lambda-\lambda_{1}\right)^{n}
$$

then operator $B$ must have nontrivial Jordan blocks otherwise the two Hamiltonian structures would be linear dependent. In this case operator $p_{11}(B)=B-\lambda_{1}$ annihilates subspace $L_{1}$ having dimension $\operatorname{dim}\left(L_{1}\right)=n_{1}<n$. Thus all operators $M_{u}, H_{u}$ and $A$ also have invariant subspace $L_{1}$. Therefore tensors $M(u, v)$ and $H(u, v)$ are reducible and the polynomials (7.1) and (7.2) are reducible too. We note that the polynomial (7.2) always has factor $\lambda$ because $\operatorname{det} H_{u} \equiv 0$. 
Obviously we have a filtration of invariant subspaces (which can coincide)

$$
L_{1} \subset L_{2} \subset \ldots \subset L_{n}=R^{n}
$$

where $L_{k}$ is annihilated by the operator

$$
p_{1 k}(B)=\left(B-\lambda_{1}\right)^{k}, \quad p_{1 k}(B)\left(L_{k}\right)=0 .
$$

Filtration (7.12) is invariant also under the operators $A, M_{u}$ and $H_{u}$ and hence it leads to a more fine factorization of the polynomials (7.1) and (7.2).

4) Suppose the operator $B=g_{1} g_{2}^{-1}$ has $n$ distinct eigenvalues that means two Hamiltonian structures are in general position. Let $e_{1}, \ldots, e_{n}$ be the basis of its (complex) eigenvectors. All operators $A, M_{u}$ and $H_{u}$ commuting with operator $B$ are diagonal in the basis on its eigenvectors. Thus all operators $A, M_{u}, M_{v}$ commute one with another and hence $H_{u}=\left[M_{u}, A\right]=0$. Therefore the Haantjes tensor vanishes: $H(u, v) \equiv 0$. The polynomial (7.1) is decomposed in this case into a product of $n$ linear factors.

Theorem 9 is proven.

II.

Corollary 5. If a system (1.1) has two Hamiltonian structures in general position and operator $A$ has $n$ real distinct eigenvalues then the system has $n$ Riemann invariants and is integrable by the generalized hodograph transformation.

Proof. The system (1.1) under these conditions has in view of the Theorem 9 zero Haantjes tensor. Therefore in view of Theorem 1 of [11] the system possesses $n$ Riemann invariants and is transformed to the diagonal form after some change of coordinates. Thus in this case the integrability by the generalized hodograph transformation follows from the Tsarev Theorem [19].

Remark 12. Bi-Hamiltonian systems were studied in many papers, where the main method to prove their integrability was based on the Magri scheme [10]. The proof of Corollary 5 is independent on the Magri approach.

Theorem 10. If a system (1.1) has two non-degenerate Hamiltonian structures and all eigenvalues $\lambda_{i}$ of the operator $B=g_{i} g_{2}^{-1}$ have multiplicities $m_{i} \leqq 2$ then the Haantjes tensor $H_{j k}^{i}=0$. If the multiplicities $m_{i} \leqq 3$ then the Haantjes tensor defines the structure of the Lie algebra in each tangent space $T_{x}\left(M^{n}\right)$. This Lie algebra is a direct sum of the commutative one $R^{k}$ and the 3-dimensional Lie subalgebras $\mathscr{A}_{3}$ with the commutators (3.14).

Proof. The generalized eigenspaces $L_{i}$ of the operator $B=g_{1} g_{2}^{-1}$ belonging to the eigenvalues $\lambda_{i}$ are invariant with respect to the operators $A$ and $H_{u}$ in view of (7.4). The Haantjes tensor $H(u, v)$ is zero on each $L_{i}$ if $\operatorname{dim} L_{i}=2$ in view of (6.47). If $\operatorname{dim} L_{i}=3$ then the Haantjes tensor $H(u, v)$ defines the structure of the Lie algebra in the $L_{i}$ with the commutators (3.14), in view of the formula (2.15) and $H_{u}=\left[M_{u}, A\right]$.

Theorem 10 is proven. 


\section{An application to the equations of gas dynamics}

We consider the equations of one-dimensional gas dynamics

$$
\begin{aligned}
v_{t} & =-v v_{x}-\frac{1}{\rho} p_{\rho} \rho_{x}-\frac{1}{\rho} p_{s} s_{x}, \\
\rho_{t} & =-\rho v_{x}-v \rho_{x}, \\
s_{t} & =-v s_{x},
\end{aligned}
$$

where $v(x, t)$ is the velocity of the gas, $\rho(x, t)$ is the density of mass and $s(x, t)$ is the density of entropy. Pressure $p$ is determined by an equation of state $p=p(\rho, s)$.

The corresponding $(1,1)$ tensor field $A_{j}^{i}$ defined on the manifold $M^{3}$ with the coordinates $v, \rho, s$ has the following eigenvalues $\lambda_{1}, \lambda_{2}, \lambda_{3}$ and eigenvectors $e_{1}, e_{2}, e_{3}$ :

$$
\begin{gathered}
\lambda_{1}=-v, \quad e_{1}=p_{s} \frac{\partial}{\partial \rho}-p_{\rho} \frac{\partial}{\partial s}, \\
\lambda_{2}=-v+\sqrt{p_{\rho}}, \quad e_{2}=\sqrt{p_{\rho}} \frac{\partial}{\partial v}-\rho \frac{\partial}{\partial \rho}, \\
\lambda_{3}=-v-\sqrt{p_{\rho}}, \quad e_{3}=\sqrt{p_{\rho}} \frac{\partial}{\partial v}+\rho \frac{\partial}{\partial \rho} .
\end{gathered}
$$

In the hyperbolic case $p_{\rho} \geqq 0$ it is easy to calculate the Haantjes tensor $H(u, v)$ in the basis of eigenvectors (8.2) using the formula (2.15). Thus we obtain:

$$
H\left(e_{1}, e_{2}\right)=h(\rho, s) e_{3}, \quad H\left(e_{1}, e_{3}\right)=h(\rho, s) e_{2}, \quad H\left(e_{2}, e_{3}\right)=0,
$$

where

$$
h(\rho, s)=p_{\rho}\left(p_{\rho} p_{\rho s}-p_{s} p_{\rho \rho}-\frac{2}{\rho} p_{\rho} p_{s}\right)=\rho^{2} p_{\rho}^{3}\left(\frac{p_{s}}{\rho^{2} p_{\rho}}\right)_{\rho} .
$$

Proposition 6. The necessary and sufficient condition for the existence of Riemann invariants for the equations of gas dynamics (8.1) is

$$
p_{\rho}=0 \quad \text { or }\left(\frac{p_{s}}{\rho^{2} p_{\rho}}\right)_{\rho}=0 .
$$

This is also the necessary condition for the existence of a Hamiltonian structure of the type (6.6) with a nondegenerate metric $g^{i j}$.

Proof. Condition (8.5) in view of (8.3), (8.4) is the condition of vanishing of the Haantjes tensor $H(u, v)$. Therefore the statement concerning the existence of Riemann invariants follows from Theorem 1 of [11].

If system (8.1) is Hamiltonian in the sense (6.6) then in view of Theorem 2 of this paper the Haantjes tensor $H(u, v)$ defines a deformation of structures of Lie algebras in the tangent bundle $T\left(M^{3}\right)$ which are simple or commutative. Lie algebras defined by the formulae (8.3) are solvable if $h(\rho, s) \neq 0$. Thus we get the necessary condition $h(\rho, s)=0$, or $(8.5)$ for the existence of a Hamiltonian structure (6.6). Proposition 6 is proven.

The operator $H_{u}$ for a tangent vector $u=x_{1} e_{1}+x_{2} e_{2}+x_{3} e_{3}$ has the form 


$$
H_{u}\left(e_{1}\right)=-h x_{3} e_{2}-h x_{2} e_{3}, \quad H_{u}\left(e_{2}\right)=h x_{1} e_{3}, \quad H_{u}\left(e_{3}\right)=h x_{1} e_{2} .
$$

The corresponding metric on the tangent space $T_{x}\left(M^{3}\right)$ is degenerate

$$
(u, u)_{H}=\operatorname{Tr}\left(H_{u} H_{u}\right)=2 h^{2} x_{1}^{2} .
$$

The algebraic manifold $V_{H} \subset T_{x}\left(M^{3}\right)$ is defined by the formula

$$
P_{H}(u)=\operatorname{det}\left(H_{u}-1\right)=h^{2} x_{1}^{2}-1=0,
$$

and thus consist of two parallel planes $x_{1}= \pm h^{-1}$.

\section{On Perturbations of the Benney Equations}

I.The Benney equations [12] have the form

$$
\begin{aligned}
& u_{i t}=-u_{i} u_{i x}-\left(\sum_{j=1}^{n} \eta_{j x}\right), \\
& \eta_{i t}=-\eta_{i} u_{i x}-u_{i} \eta_{i x} .
\end{aligned}
$$

On the corresponding manifold $M^{2 n}$ we consider the local coordinates $u_{1}, \ldots, u_{n}$, $\eta_{1}, \ldots, \eta_{n}$. In the tangent space $T_{x}\left(M^{2 n}\right)$ we have a basis of $2 n$ vectors

$$
e_{i}=\frac{\partial}{\partial u_{i}}, \quad g_{i}=\frac{\partial}{\partial \eta_{i}}, \quad i=1, \ldots, n .
$$

The operator tensor field $A_{j}^{i}$ defined by the Benney system (9.1) has the form

$$
A e_{i}=-u_{i} e_{i}-\eta_{i} g_{i}, \quad A g_{i}=-\left(e_{1}+\ldots+e_{n}\right)-u_{i} g_{i} .
$$

A direct calculation of the Nijenhuis tensor $N(v, w)(2.1)$ leads to the formulae

$$
\begin{gathered}
N\left(e_{i}, e_{j}\right)=0, N\left(g_{i}, g_{j}\right)=g_{j}-g_{i}, \\
N\left(e_{i}, g_{j}\right)=-N\left(g_{j}, e_{i}\right)=-e_{i} .
\end{gathered}
$$

Hence for any two tangent vectors

$$
v=\sum_{i=1}^{n}\left(x_{i} e_{i}+y_{i} g_{i}\right), \quad \alpha=\sum_{i=1}^{n}\left(\alpha_{i} e_{i}+\beta_{i} g_{i}\right),
$$

we have

$$
\begin{gathered}
N_{v} e_{i}=N\left(v, e_{i}\right)=\omega(v) e_{i}, \quad N_{v} g_{j}=\omega(v) g_{j}-v, \\
N_{v} \alpha=N(v, \alpha)=\omega(v) \alpha-\omega(\alpha) v .
\end{gathered}
$$

Here $\omega$ is the differential 1 -form

$$
\omega(v)=y_{1}+\ldots+y_{n}, \quad \omega(\alpha)=\beta_{1}+\ldots+\beta_{n} .
$$

From (9.3) we get

$$
A v=\sum_{i=1}^{n}\left(-x_{i} u_{i}-\omega(v)\right) e_{i}-\sum_{i=1}^{n}\left(x_{i} \eta_{i}+y_{i} u_{i}\right) g_{i} .
$$


From (9.7) and (9.9) we obtain

$$
\begin{gathered}
M_{v} \alpha=\left(N_{A v}-A N_{v}\right) \alpha=-\omega(v) A \alpha-\tilde{\omega}(v) \alpha, \\
M_{v}=-\omega(v) A-\tilde{\omega}(v) 1
\end{gathered}
$$

where

$$
\tilde{\omega}(v)=\sum_{i=1}^{n}\left(x_{i} \eta_{i}-y_{i} u_{i}\right) .
$$

The Haantjes tensor $H(v, w)$ for the Benney system (9.1) is identically equal to zero, that follows from (9.11) and (2.6),

$$
H_{v}=\left[M_{v}, A\right]=0 \text {. }
$$

This gives in view of Theorem 1 of the work [11] another proof of the existence of the Riemann invariants for the Benney equations.

II. Let us show that the Nijenhuis tensor (9.7) for the Benney equations defines the structure of the Lie algebra in each tangent space $T_{x}\left(M^{2 n}\right)$. Indeed, let $L^{2 n-1}$ be the hyperplane of the tangent vectors, satisfying the condition $\omega(v)=0$, and $g$ be the tangent vector

$$
g=\frac{1}{n}\left(g_{1}+\ldots+g_{n}\right) .
$$

Obviously $\omega(g)=1$. In view of (9.7) for any two vectors $v, w \in L^{2 n-1}$ we have

$$
N(v, w)=0, \quad N(g, v)=v .
$$

The Jacobi identity for any three tangent vectors $u, v, w \in T_{x}\left(M^{2 n}\right)$

$$
N(N(u, v), w)+N(N(v, w), u)+N(N(w, u), v)=0
$$

follows easily from the formulae (9.15). Thus each tangent space $T_{x}\left(M^{2 n}\right)$ has the structure of the solvable Lie algebra $\mathscr{A}$, determined by the Nijenhuis tensor (9.7). The hyperplane $L^{2 n-1}$ is the maximal commutative ideal:

$$
\left[L^{2 n-1}, L^{2 n-1}\right]=0, \quad[\mathscr{A}, \mathscr{A}]=L^{2 n-1} .
$$

Obviously the structure of the Lie Algebra $\mathscr{A}$ in $T_{x}\left(M^{2 n}\right)$ does not depend on the point $x \in M^{2 n}$.

The invariant scalar product $(u, v)_{N}(2.8)$ coincides with the Cartan-Killing form

$$
(u, v)_{N}=\operatorname{Tr}\left(N_{u} N_{v}\right)=(2 n-1) \omega(u) \omega(v) .
$$

Thus we have

$$
\left(L^{2 n-1}, L^{2 n-1}\right)_{N}=0, \quad\left(L^{2 n-1}, g\right)_{N}=0, \quad(g, g)_{N}=2 n-1 .
$$

The algebraic manifold $V_{N} \in T_{x}\left(M^{2 n}\right)$ for the Benney system is determined by the equation

$$
P_{N}(v)=\operatorname{det}\left(N_{v}-1\right)=(\omega(v)-1)^{2 n-1}=0 .
$$

Thus the manifold $V_{N}$ is the hyperplane $\omega(v)=1$, parallel to the plane $L^{2 n-1}$.

Plane $L^{2 n-1}$ is the set $Z_{N}$ of all $N$-nilpotent vectors, see (5.22)-(5.24). 
III. We consider the following natural perturbations of the Benney equations:

$$
\begin{aligned}
& u_{i t}=-u_{i} u_{i x}-\sum_{k=1}^{n} f_{k}\left(\eta_{k}\right) \eta_{k x}, \\
& \eta_{t t}=-\eta_{i} u_{i x}-u_{i} \eta_{i x}
\end{aligned}
$$

where $f_{k}\left(\eta_{k}\right)$ are arbitrary smooth functions. System (9.21) for $f_{k}\left(\eta_{k}\right)=$ const is equivalent to the Benney system (9.1).

The corresponding to $(9.21)(1,1)$ tensor field $A_{j}^{i}$ has the form

$$
A e_{i}=-u_{i} e_{i}-\eta_{i} g_{i}, \quad A g_{i}=-f_{i} E-u_{i} g_{i},
$$

where

$$
e_{i}=\frac{\partial}{\partial u_{i}}, \quad g_{i}=\frac{\partial}{\partial \eta_{i}}, \quad E=e_{1}+\ldots+e_{n} .
$$

For any tangent vector $v(9.5)$ we have

$$
A v=-\sum_{k=1}^{n} x_{k} u_{k} e_{k}-\sum_{k=1}^{n}\left(x_{k} \eta_{k}+y_{k} u_{k}\right) g_{k}-\omega(v) E,
$$

where $\omega(v)$ is the differential 1 -form

$$
\omega(v)=\sum_{k=1}^{n} f_{k}\left(\eta_{k}\right) y_{k}
$$

The Nijenhuis tensor for the system (9.21) has the form

$$
\begin{aligned}
& N\left(e_{i}, e_{j}\right)=0, \quad N\left(g_{i}, g_{j}\right)=f_{i} g_{j}-f_{j} g_{i}, \\
& N\left(g_{j}, e_{i}\right)=-N\left(e_{i}, g_{j}\right)=f_{j} e_{i}-\eta_{j} \dot{f}_{j} \delta_{j}^{i} E, \quad \dot{f}_{j}=\frac{d f_{i}\left(\eta_{j}\right)}{d \eta_{j}} .
\end{aligned}
$$

Hence for any two tangent vectors $v, \alpha(9.5)$ we obtain

$$
N(v, \alpha)=N_{0}(v, \alpha)+\omega_{0}(v, \alpha) E,
$$

where

$$
\begin{gathered}
N_{0}(v, \alpha)=\omega(v) \alpha-\omega(\alpha) v \\
\omega_{0}(v, \alpha)=\sum_{k=1}^{n}\left(x_{k} \beta_{k}-y_{k} \alpha_{k}\right) \eta_{k} \dot{f}_{k} .
\end{gathered}
$$

Tensor $N_{0}(v, \alpha)(9.28)$ is analogous to (9.7) and therefore satisfies the Jacobi identity $(9.16)$. Tensor $N(v, \alpha)(9.27)$ satisfies the Jacobi identity only if $\omega_{0}(v, \alpha) \equiv 0$, or $f_{k}\left(\eta_{k}\right)=$ const, i.e., only for the Benney system (9.1).

From (9.27) and (9.24) we get

$$
\begin{aligned}
M_{v} \alpha & =\left(N_{A v}-A N_{v}\right) \alpha \\
& =\omega_{0}(A v, \alpha) E-\omega_{0}(v, \alpha) A E-\omega(v) A \alpha-\tilde{\omega}(v) \alpha,
\end{aligned}
$$

where

$$
\tilde{\omega}(v)=\sum_{k=1}^{n} f_{k}\left(x_{k} \eta_{k}-y_{k} u_{k}\right)
$$

Hence the formula for the operators $H_{v}$ follows 


$$
H_{v} \alpha=\left[M_{v}, A\right] \alpha=\omega_{0}(v, \alpha) A^{2} E+\omega_{1}(v, \alpha) A E+\omega_{2}(v, \alpha) E
$$

where

$$
\begin{gathered}
\omega_{1}(u, \alpha)=\omega_{0}(A \alpha, u)+\omega_{0}(\alpha, A u), \\
\omega_{2}(u, \alpha)=\omega_{0}(A u, A \alpha) .
\end{gathered}
$$

Thus we get that operators $H_{v}$ for the system (9.21) are not zero and transform a tangent space $T_{x}\left(M^{2 n}\right)$ into 3-dimensional subspace generated by vectors

$$
E, A E, A^{2} E \text {. }
$$

Vectors (9.34) are linear independent and for a general vector $v$ three 1-forms defined by the formulae (9.29), (9.32) and (9.33)

$$
\omega_{0}(v, \alpha), \omega_{1}(v, \alpha), \omega_{2}(v, \alpha)
$$

are linear independent too. Thus the dimension of the image $H_{v}\left(T_{x}\left(M^{2 n}\right)\right)$ for the general vector $v$ is odd. Therefore in view of the Theorem 3 of this work (the necessary condition 3 ) is not fulfilled) and Theorem 1 of the work [11] we obtain the following result.

Proposition 7. Any perturbation (9.21) of the Benney equations (9.1) with nonconstant functions $f_{k}\left(\eta_{k}\right)$ has no Riemann invariants and has no Hamiltonian structure of the non-degenerate type (6.6).

$I V$. Let $K \subset T_{x}\left(M^{2 n}\right)$ be the kernel of the operator $H_{v}$ and $h_{1}, \ldots, h_{2 n-3}$ be a basis in $K$. Operator $H_{v}$ in the basis of $2 n$ vectors

$$
E, A E, A^{2} E, h_{1}, \ldots, h_{2 n-3}
$$

has nonzero entries only in the up-left $3 \times 3$ block. Therefore the polynomial

$$
P_{H}(v)=\operatorname{det}\left(H_{v}-1\right)
$$

has the form

$$
P_{H}(v)=-\operatorname{det}\left[\begin{array}{llc}
\omega_{2}(v, E)-1 & \omega_{2}(v, A E) & \omega_{2}\left(v, A^{2} E\right) \\
\omega_{1}(v, E) & \omega_{1}(v, A E)-1 & \omega_{1}\left(v, A^{2} E\right) \\
\omega_{0}(v, E) & \omega_{0}(v, A E) & \omega_{0}\left(v, A^{2} E\right)-1
\end{array}\right] .
$$

This polynomial has degree 3 and the corresponding algebraic manifold $V_{H} \subset$ $T_{x}\left(M^{2 n}\right)$, determined by the equation $P_{H}(v)=0$, is not invariant under the reflection $v \rightarrow-v$. Thus the necessary conditions 1 ) and 2) from Theorem 3 for the existence of a Hamiltonian structure are not fulfilled for the perturbation of the Benney equations (9.21).

\section{An Application to the Certain Matrix Partial Differential Equations}

I. We consider for an arbitrary Lie algebra $\mathscr{A}$ the partial differential equation for a vector function $a(t, x) \in \mathscr{A}$ having the Lax form

$$
a_{t}=\left[a, a_{x}\right] \text {. }
$$


The Casimir functions $f(a)$, constant on the orbits of the adjoint representation of the corresponding Lie group $G$, are the pointwise first integrals of (10.1): $f(a(t, x))_{t}=0$.

The manifold $M^{n}$ corresponding to the system (10.1) coincides with the Lie algebra $\mathscr{A}$. The corresponding operator tensor field is $A=\operatorname{ad}_{a}$. Let $u, v \in T_{a}(\mathscr{A})$ be two tangent vectors in a point $a \in A$. The operator tensor field $A$ is skew-symmetric with respect to the Cartan-Killing form

$$
(u, v)=\operatorname{Tr}\left(\operatorname{ad}_{u} \operatorname{ad}_{v}\right),(A u, v)+(u, A v)=0 .
$$

We consider the constant extensions $\tilde{u}, \tilde{v}$ as the vector fields on the manifold $M^{n}=$ $\mathscr{A}$. The Nijenhuis tensor is determined by the formula

$$
N(u, v)=A^{2}[\tilde{u}, \tilde{v}]_{g}+[A \tilde{u}, A \tilde{v}]_{g}-A\left([A \tilde{u}, \tilde{v}]_{g}+[\tilde{u}, A \tilde{v}]_{g}\right),
$$

where $[\alpha, \beta]_{g}$ is the geometric commutator of the vector fields $\alpha, \beta$.

Let $c_{i j}^{k}$ be structure constants of Lie algebra $\mathscr{A}$ in the basis

$$
e_{1}=\frac{\partial}{\partial a^{1}}, \ldots, e_{n}=\frac{\partial}{\partial a^{n}}, \quad\left[e_{i}, e_{j}\right]=C_{i j}^{k} e_{k} .
$$

Then we have

$$
A \tilde{u}=a d_{a} \tilde{u}=\sum_{i, j, k}^{n} C_{i j}^{k} a^{i} u^{j} \frac{\partial}{\partial a^{k}}, A \tilde{v}=\sum_{i, j, s}^{n} C_{i j}^{s} a^{i} v^{j} \frac{\partial}{\partial a^{s}},
$$

and the formulae follow

$$
\begin{aligned}
& {[A \tilde{u}, A \tilde{v}]_{g}=[a,[u, v]],[\tilde{u}, \tilde{v}]_{g}=0,} \\
& {[A \tilde{u}, \tilde{v}]_{g}=[u, v],[\tilde{u}, A \tilde{v}]_{g}=[u, v] .}
\end{aligned}
$$

substituting these formulae into (10.3), we obtain

$$
N(u, v)=-[a,[u, v]], N_{u}=-\operatorname{ad}_{a} \operatorname{ad}_{u} .
$$

Hence we obtain for the operators $M_{u}$ :

$$
M_{u}=N_{A u}-A N_{u}=N_{[a, u]}+\operatorname{ad}_{a}^{2} \operatorname{ad}_{u}=\operatorname{ad}_{a} \operatorname{ad}_{u} \operatorname{ad}_{a} .
$$

Therefore for the Haantjes operators $H_{u}$ we get the expression

$$
H_{u}=\left[M_{u}, A\right]=\operatorname{ad}_{a} \operatorname{ad}_{[u, a]} \operatorname{ad}_{a} .
$$

From the formulae (10.2) (10.7) and (10.8) we obtain that all operators $M_{u}, H_{v}$ and $A$ are skew-symmetric with respect to the Cartan-Killing form (10.2).

Proposition 8. Equation (10.1) for any simple Lie algebra $\mathscr{A}$ considered as a system of partial differential equations has no Riemann invariants and no Hamiltonian structure of the nondegenerate type (6.6).

Proof. If a system (10.1) for some Lie algebra $\mathscr{A}$ is Hamiltonian in the sense of (6.6), then all operators $M_{u}$ and $A$ are symmetric with respect to the bilinear form (6.9) and all operators $H_{u}$ are skew-symmetric. In this case analogously to Sect. 7 the non-degenerate operators $B_{a}$ have to exist that anticommute with all operators $M_{u}$ (10.7) and $A=\operatorname{ad}_{a}$ and commute with all operators $H_{u}$ (10.8). For a simple Lie 
algebra $\mathscr{A}$ such operators $B_{a}$ do not exist in view of the explicit formulae (10.6)(10.8). Therefore Eq. (10.1) for any simple Lie algebra $\mathscr{A}$ is not Hamiltonian.

The nonexistence of the Riemann invariants follows from the nonvanishing of the Haantjes tensor $H(u, v)(10.8)$ and from Theorem 1 of [11]. Proposition 8 is proven.

II. Let us consider an equation for a matrix $a(t, x)$ :

$$
a_{t}=\lambda a a_{x}+\mu a_{x} a,
$$

where $\lambda$ and $\mu$ are some constants. Matrix entries $a^{i j}$ are coordinates in the space $M^{N}$ of all matrices, $N=n^{2}$. Tangent vectors $e_{i j}=\partial / \partial a^{i j}$ form a basis in a tangent space $T_{a}\left(M^{N}\right)$ which is naturally identified with the space of matrices $M^{N}$. Equation (10.9) in the coordinates $a^{i j}$ has the form

$$
a_{t}^{i j}=\lambda a^{i \alpha} a_{x}^{\alpha j}+\mu a_{x}^{i \alpha} a^{\alpha j} .
$$

The corresponding operator $A$ has the entries

$$
A_{k s}^{i j}=\lambda a^{i k} \delta_{s}^{j}+\mu \delta_{k}^{i} a^{s j}, \quad A v=\lambda a v+\mu v a .
$$

A direct calculation by the formula (2.2) leads to an expression for the Nijenhuis tensor

$$
N\left(e_{i j}, e_{k s}\right)=\lambda \mu\left(\delta_{i}^{s}\left(a^{j \alpha} e_{k \alpha}-a^{\alpha k} e_{\alpha j}\right)+\delta_{j}^{k}\left(a^{\alpha i} e_{\alpha s}-a^{s \alpha} e_{i \alpha}\right)\right) .
$$

Hence for any two tangent vectors $u, v \in T_{a}\left(M^{N}\right)$ we obtain

$$
N(u, v)=\lambda \mu[a,[u, v]] .
$$

That provides for $\lambda=1$ and $\mu=-1$ the second proof of the formula (10.6).

Proposition 9. Matrix equations

$$
\begin{aligned}
& a_{t}=P(a) a_{x}, \\
& a_{t}=a_{x} P(a),
\end{aligned}
$$

where $P(a)$ is an arbitrary analytic matrix function split into $n$ noninteracting subsystems of partial differential equations in a neighbourhood of any matrix a with real and distinct eigenvalues.

Proof. We prove at first that the Nijenhuis tensor vanishes for Eqs. (10.13) and (10.14). Indeed, we get from (10.12) that equations

$$
\begin{aligned}
& a_{t}=a a_{x}, \\
& a_{t}=a_{x} a
\end{aligned}
$$

have zero Nijenhuis tensor, because $\lambda \mu=0$. If a matrix $a$ has diagonal real Jordan normal form, then operators $A$ (10.11), corresponding to Eqs. (10.15), (10.16) also have diagonal Jordan normal forms, because $A v=a v$ or $A v=v a$. Operator tensor fields, corresponding to Eqs. (10.13) and (10.14) are $P(A)$. Let $e_{1}, \ldots, e_{N}$ be eigenvectors of an operator $A$ with diagonal Jordan normal form and $\lambda_{1}, \ldots, \lambda_{N}$ be the corresponding eigenvalues. Operator $P(A)$ has the same eigenvectors $e_{1}, \ldots, e_{N}$ with eigenvalues $P\left(\lambda_{1}\right), \ldots, P\left(\lambda_{N}\right)$. From the Nijenhuis formula 


$$
N\left(e_{i}, e_{j}\right)=\left(A-\lambda_{i}\right)\left(A-\lambda_{j}\right)\left[e_{i}, e_{j}\right]+\left(\lambda_{i}-\lambda_{j}\right)\left(e_{i}\left(\lambda_{j}\right) e_{j}+e_{j}\left(\lambda_{i}\right) e_{i}\right)
$$

it follows that if the Nijenhuis tensor for the operator $A$ is equal to zero then it is equal to zero for all operators $P(A)$ as well.

Eigenvalues of the operators $P(A)$ corresponding to Eqs. (10.13), (10.14) are equal to $P\left(\lambda_{i}\right)$ where $\lambda_{i}$ form $n$ eigenvalues of the matrix $a$ and have multiplicities $n$. Therefore from the results of the work [16] in view of the vanishing of the Nijenhuis tensor we obtain that Eqs. (10.13), (10.14) split into $n$ non-interacting subsystems of $n$ equations (in each subsystem). These subsystems are tangent to $n$-dimensional eigenspaces of the operator $P(A)$ and have the form

$$
\lambda_{i t}=P\left(\lambda_{i}\right) \lambda_{i x}, \quad u_{i k t}=P\left(\lambda_{i}\right) u_{i k x}, \quad k=1, \ldots, n-1 .
$$

The arising $n^{2}$ functions $\lambda_{1}, \ldots, \lambda_{n}, u_{i k}, 1 \leqq i \leqq n, 1 \leqq k \leqq n-1$ form another system of coordinates in the space of matrices $M^{N}$. Proposition 9 is proven.

III. We consider the matrix equation

$$
a_{t}=a a_{x} a .
$$

For the entries $a^{i j}$ of the matrix $a$ we get the equations

$$
a_{t}^{i j}=a^{i \alpha} a_{x}^{\alpha \beta} a^{\beta j} .
$$

The corresponding operator $A$ has entries

$$
A_{k s}^{i j}=a^{i k} a^{s j}, \quad A v=a v a .
$$

The operator $A$ is symmetric with respect to the bilinear form

$$
(u, v)=\operatorname{Tr}(u v), \quad(A u, v)=(u, A v) .
$$

A direct calculation by the formula (2.2) leads to the following expression for the Nijenhuis tensor:

$$
N\left(e_{i j}, e_{k s}\right)=\left(\delta_{s}^{i} a^{j \beta} a^{\beta \delta} a^{\gamma k}+\delta_{k}^{j} a^{\gamma \alpha} a^{\alpha i} a^{s \delta}-\delta_{j}^{k} a^{s \beta} a^{\beta \delta} a^{\gamma i}-\delta_{i}^{s} a^{\gamma \alpha} a^{\alpha k} a^{j \delta}\right) e_{\gamma \delta} .
$$

Hence for any two tangent vectors $u, v \in T_{a}\left(M^{N}\right)$ we get

$$
N(u, v)=a[a,[u, v]] a .
$$

Thus we have

$$
N_{u}=\operatorname{Aad}_{a} \operatorname{ad}_{u} .
$$

For the operators $M_{u}$ we get the formula

$$
M_{u}=N_{A u}-A N_{u}=A \operatorname{ad}_{a}\left(\operatorname{ad}_{a u a}-A \operatorname{ad}_{u}\right) .
$$

Therefore for the Haantjes tensor $H_{u}$ we obtain

$$
H_{u}=\left[M_{u}, A\right]=A \operatorname{ad}_{a}\left[\operatorname{ad}_{a u a}-A \operatorname{ad}_{u}, A\right]=A^{2} \operatorname{ad}_{a} \operatorname{ad}_{[u, a]} \operatorname{ad}_{a} .
$$

Thus we get that the Haantjes tensor (10.27) is not equal to zero and so Eq. (10.19) as well as Eqs. (10.1) has no Riemann invariants.

Acknowledgement. This paper was presented on May 17, 1993 in the Director's Seminar at the Fields Institute for Research in Mathematical Sciences. The author is grateful to Jerrold Marsden for the invitation and for a useful discussion. 


\section{References}

1. Riemann, G.F.B.: Über die Fortpflanzung ebener Luftwellen von endlicher Schwingungsweite. Abhandl. Konigl. Ges. Wiss. Göttingen, v. 8, 1860

2. Courant, R., Hilbert, D.: Methods of mathematical physics, v. II. New York: Interscience Publishers, 1962

3. Lax, P.D.: Weak solutions of nonlinear hyperbolic equations and their numerical computation. Commun. Pure Appl. Math. 7, 159-193 (1954)

4. Glimm, J.: Solutions in the large for nonlinear hyperbolic systems of equations. Commun. Pure Appl. Math. 18, 697-715 (1965)

5. Nijenhuis, A.: $X_{n-1}$-forming sets of eigenvectors. Proc. Kon. Ned. Akad. Amsterdam 54, 200 $212(1951)$

6. Haantjes, J.: On $X_{m}$-forming sets of eigenvectors. Proc. Kon. Ned. Akad. Amsterdam 58, 158-162 (1955)

7. Dubrovin, B.A., Novikov, S.P.: On Poisson brackets of hydrodynamic type. Sov. Math. Dokl. 30, 651-654 (1984)

8. Novikov, S.P.: The geometry of conservative systems of hydrodynamic type. The method of averaging for field-theoretical systems. Russ. Math. Surv. 40, N4, 85-98 (1985)

9. Dubrovin, B.A., Novikov, S.P.: Hydrodynamics of weakly deformed soliton lattices. Differential geometry and Hamiltonian theory. Russ. Math. Surv. 44, N6, 35-124 (1989)

10. Magri, F.: A simple model of an integrable Hamiltonian system. J. Math. Phys. 19, 1156-1162 (1978)

11. Bogoyavlenskij, O.I.: Existence of Riemann invariants and Hamiltonian structures. C.R. Math. Rep. Acad. Sci. Canada 15, 143-148 (1993)

12. Benney, D.J.: Some properties of long non-linear waves. Stud. Appl. Math. 52, 45-50 (1973)

13. Whitham, G.B.: Non-linear dispersive waves. Proc. Royal Soc. Lond. Ser. A 139, 283-291 (1965)

14. Ercolani, N., Forest, M.G., McLaughlin, D.W., Montgomery, R.: Hamiltonian structure of the modulation equations of a Sine-Gordon wavetrain. Duke Math. J. 55, 949-983 (1987)

15. Flaschka, H., Forest, M.G., McLaughlin, D.W.: Multiphase averaging and the inverse spectral solution of the Korteweg-de Vries equation. Commun. Pure Appl. Math. 33, 739-784 (1980)

16. Stone, A.P.: Generalized conservation laws. Proc. of Am. Math. Soc. 18, 868-873 (1967)

17. Weyl, H.: The classical groups, their invariants and representations. Princeton, NJ: Princeton University Press, 1946

18. Eisenhart, L.P.: Riemannian geometry. Princeton, NJ: Princeton University Press, 1964

19. Tsarev, S.P.: On Poisson brackets and Hamiltonian systems of hydrodynamic type. Sov. Math. Dokl. 31, 488-491 (1985)

20. Mokhov, O.I., Ferapontov, E.V.: Non-local Hamiltonian operators of hydrodynamic type related to metrices of constant curvature. Russ. Math. Surv. 45, N2, 218-219 (1990)

Communicated by H. Araki

\section{Note added in proof}

\section{Necessary Conditions for Existence of a Non-Local Non-Degenerate Hamiltonian Structure}

I. Let us consider a $(1,1)$ tensor

$$
A_{j}^{i}(u)=g^{i \alpha} \nabla_{\alpha} \nabla_{j} f(u)+K f(u) \delta_{J}^{i},
$$

where $g_{l J}$ is a non-degenerate metric of constant curvature $K, f(u)$ is a smooth function and $\nabla_{J}$ are operators of covariant differentiation with respect to the metric $g_{i j}$. The $(1,1)$ tensors $A_{j}^{l}(u)$ (11.1) describe systems of partial differential equations (1.1) having non-local $(K \neq 0)$ [20] or local $(K=0)[7-9]$ non-degenerate Hamiltonian structures. 
Theorem 11. If a system (1.1) has a non-local or local non-degenerate Hamiltonian structure then the Nijenhuis tensor $N_{A}(u, v)$ satisfies the algebraic identities $(6.42)-(6.44)$ for the $(1,1)$ tensor $A_{j}^{i}$ (11.1). The corresponding operators $H_{u}(2.5)$ are skew-symmetric with respect to the bilinear form defined by the metric $g_{i j}$ :

$$
\left(H_{u} v, w\right)_{g}=-\left(v, H_{u} w\right)_{g},(v, w)_{g}=g_{\alpha \beta} v^{\alpha} w^{\beta} .
$$

The proof follows by a direct calculation using the classical formulae of differential geometry for the metric of constant curvature.

Corollary 6. Theorems 3-10 hold true for the systems (1.1) possessing non-local and nondegenerate Hamiltonian structures.

Indeed, Theorems 3-10 are direct consequences of the identity (6.14) and the algebraic identities (6.42)-(6.44). These identities hold true for the non-local Hamiltonian structures as well.

II. Let us assume that system (1.1) is hyperbolic. Then tensor $A_{j}^{l}(x)(11.1)$ has real and distinct eigenvalues $\lambda_{1}(x), \ldots, \lambda_{n}(x)$ corresponding to the eigenvectors $e_{1}(x), \ldots, e_{n}(x)$. Their commutator relations have the form

$$
\left[e_{i}(x), e_{j}(x)\right]=\sum_{k=1}^{n} C_{i j}^{k}(x) e_{k}(x) .
$$

Theorem 12. If a system (1.1) possesses a non-local or local non-degenerate Hamiltonian structure then the following necessary conditions are satisfied:

1) For any $p+1$ distinct indices $\alpha, i, \ldots, \ell$ the following two products are equal

$$
C_{\alpha i}^{j} C_{\alpha j}^{k} \ldots C_{\alpha m}^{\ell} C_{\alpha \ell}^{i}=(-1)^{p} C_{\alpha j}^{i} C_{\alpha k}^{j} \ldots C_{\alpha \ell}^{m} C_{\alpha i}^{\ell} .
$$

2) For any $p$ distinct indices $i, j, \ldots, \ell$ and $p$ arbitrary indices $\alpha, \beta, \ldots, \sigma$ different from $i, j, \ldots, \ell$ the following two products are equal

$$
D_{\alpha i}^{J} D_{\beta J}^{k} \ldots D_{\gamma m}^{\ell} D_{\sigma \ell}^{i}=(-1)^{p} D_{\alpha j}^{i} D_{\beta k}^{j} \ldots D_{\gamma \ell}^{m} D_{\sigma i}^{\ell}, D_{\alpha i}^{J}=\frac{C_{\alpha i}^{j}}{\left(\lambda_{\alpha}-\lambda_{i}\right)^{2}} .
$$

Proof. The metric $g_{i j}$ is diagonal in the basis of eigenvalues

$$
\left(e_{i}(x), e_{j}(x)\right)_{g}=q_{i}(x) \delta_{j}^{i},
$$

because the $(1,1)$ tensor $A_{j}^{l}(x)(11.1)$ is symmetric with respect to the metric $g_{i j}(x)$. The formulae (2.15), (11.2) and (11.3) imply

$$
\frac{C_{\alpha i}^{j}}{\left(\lambda_{\alpha}-\lambda_{i}\right)^{2}} q_{j}=-\frac{C_{\alpha j}^{i}}{\left(\lambda_{\alpha}-\lambda_{j}\right)^{2}} q_{i}
$$

Multiplying the formulae (11.7) for $p$ indices $\alpha, \beta, \ldots, \delta$ and $p$ pairs of indices $(i, j),(j, k), \ldots,(\ell, i)$ we obtain the equalities (11.4) and (11.5).

III. Symmetricity of the $(1,1)$ tensor $A_{j}^{i}(x)$ yields

$$
(B(A) u, v)_{g}=(u, B(A) v)_{g}, B(A, x)=\sum_{m=0}^{k} b_{m}(x) A^{m}(x)
$$

for an arbitrary polynomial $B(A, x)$. We define a polynomial on the tangent bundle $T\left(M^{n}\right)$

$$
P_{B}(u, \lambda)=\operatorname{det}\left(B(A) H_{u}-\lambda\right)
$$

and a fibration of algebraic varieties $V_{B} \subset T\left(M^{n}\right)$ defined by the equation $P_{B}(u, 1)=0$. 
Theorem 13. If a system (1.1) possesses a non-local or local non-degenerate Hamiltonian structure then the following necessary conditions are satisfied in any point $x \in M^{n}$ and for any tangent vector $u \in T_{x}\left(M^{n}\right)$ and for an arbitrary polynomial $B(A, x)(11.8)$ :

1) The polynomial $P_{B}(u, \lambda)(11.9)$ is even with respect to $u$ and is even or odd with respect to $\lambda$ whenever $n$ is even or odd.

2) The fibration of the algebraic varieties $V_{B} \subset T\left(M^{n}\right)$ is invariant with respect to the involution $u \rightarrow-u$.

3) The set of eigenvalues of the operator $B(A) H_{u}$ is invariant with respect to the involution $\lambda \rightarrow-\lambda$. Lemmas.

Proof of Theorem 13 follows from the identities (11.2) and (11.8) and the following two

Lemma 4. The characteristic polynomials coincide for the operators $B H$ and $H B$.

Lemma 5. If an operator $B$ is symmetric with respect to a non-degenerate bilinear form (see(11.8)) and an operator $H$ is skew-symmetric (11.2) then the set of eigenvalues of the operators $B H$ and $H B$ is invariant with respect to the involution $\lambda \rightarrow-\lambda$ along with their multiplicities.

Proof. The relations (11.8) and (11.2) imply the matrix equations

$$
B g=g B^{t}, H g=-g H^{t}, g^{-1} B H g=-(H B)^{t} .
$$

Using the last formula we obtain for the characteristic polynomial

$$
\begin{aligned}
P_{B H}(\lambda) & =\operatorname{det}(B H-\lambda)=\operatorname{det}\left(g^{-1} B H g-\lambda\right)=\operatorname{det}\left(-(H B)^{t}-\lambda\right) \\
& =(-1)^{n} \operatorname{det}(H B+\lambda)=(-1)^{n} P_{H B}(-\lambda) .
\end{aligned}
$$

Applying Lemma 4 we derive the equality

$$
P_{B H}(\lambda)=(-1)^{n} P_{B H}(-\lambda),
$$

that proves Lemma 5 .

Theorem 14. If two operators $P$ and $H$ are skew-symmetric with respect to a non-degenerate bilinear form (see (11.2)) then all non-zero eigenvalues of the operator PH have even multiplicites.

Proof. We first assume that $P=B H B$ where $B$ is a symmetric operator (see (11.8)). Then $P H=$ $(B H)^{2}$. The set of eigenvalues of the operator $B H$ is symmetric with respect to the involution $\lambda \rightarrow-\lambda$ along with their multiplicities (Lemma 5). Therefore all non-zero eigenvalues of the operator $(B H)^{2}$ have even multiplicities. Thus Theorem 14 is proven for the pairs $B H B, H$. For an arbitrary skew-symmetric pair $P, H$ Theorem 14 follows by the continuity arguments because the set of pairs $B H B, H$ is open and dense in the set of all skew-symmetric pairs $P, H$.

Corollary 7. If a system (1.1) possesses a non-local or local non-degenerate Hamiltonian structure then non-zero eigenvalues of the operators $H_{u}^{k} H_{v}^{m}$ have even multiplicities when $k$ and $m$ are odd and are symmetric with respect to the involution $\lambda \rightarrow-\lambda$ when $k+m$ is odd.

The methods of this paper lead to new geometric and algebraic constructions which will be published elsewhere. 
